\title{
Strange and charm baryon masses with two flavors of dynamical twisted mass fermions
}

\author{
C. Alexandrou, ${ }^{1,2}$ J. Carbonell,${ }^{3}$ D. Christaras, ${ }^{1}$ V. Drach, ${ }^{4}$ M. Gravina, ${ }^{1}$ and M. Papinutto ${ }^{5,6}$ \\ ${ }^{1}$ Department of Physics, University of Cyprus, P.O. Box 20537, 1678 Nicosia, Cyprus \\ ${ }^{2}$ Computation-based Science and Technology Research Center, Cyprus Institute, 20 Kavafi Street, Nicosia 2121, Cyprus \\ ${ }^{3}$ CEA-Saclay, IRFU/Service de Physique Nucléaire, 91191 Gif-sur-Yvette, France \\ ${ }^{4}$ NIC, DESY, Platanenallee 6, D-15738 Zeuthen, Germany \\ ${ }^{5}$ Laboratoire de Physique Subatomique et Cosmologie, UJF/CNRS/IN2P3, 53 avenue des Martyrs, 38026 Grenoble, France \\ ${ }^{6}$ Departamento de Física Teórica and Instituto de Física Teórica UAM/CSIC, \\ Universidad Autónoma de Madrid, Cantoblanco, E-28049 Madrid, Spain
}

(Received 1 June 2012; published 3 December 2012)

\begin{abstract}
The masses of the low-lying strange and charm baryons are evaluated using two degenerate flavors of twisted mass sea quarks for pion masses in the range of about 260 to $450 \mathrm{MeV}$. The strange and charm valence quark masses are tuned to reproduce the masses of the kaon and $D$ meson at the physical point. The tree-level Symanzik improved gauge action is employed. We use three values of the lattice spacing, corresponding to $\beta=3.9, \beta=4.05$ and $\beta=4.2$, with $r_{0} / a=5.22(2), r_{0} / a=6.61(3)$ and $r_{0} / a=8.31(5)$, respectively. We examine the dependence of the strange and charm baryons on the lattice spacing and the strange and charm quark masses. The pion mass dependence is studied and physical results are obtained using heavy-baryon chiral perturbation theory to extrapolate to the physical point.
\end{abstract}

DOI: 10.1103/PhysRevD.86.114501

PACS numbers: 11.15.Ha, 12.38.-t, 12.38.Aw, 12.38.Gc

\section{INTRODUCTION}

Lattice QCD simulations with two light degenerate sea quarks $\left(N_{f}=2\right)$, as well as those with a strange sea quark $\left(N_{f}=2+1\right)$ close to physical values of the pion mass, are being carried out. Masses of low-lying hadrons are primary quantities that can be extracted using these simulations. Comparing the lattice and experimental values provides a check of lattice discretization effects. Such a comparison is necessary before one can use the lattice approach to study hadron structure. The European Twisted Mass (ETM) Collaboration has generated a number of $N_{f}=2$ ensembles at four values of the lattice spacing, ranging from $0.1 \mathrm{fm}$ to about $0.05 \mathrm{fm}$, at several values of the light sea quark mass, and for several physical volumes with maximally twisted mass fermions. We will use ensembles generated at the three smallest lattice spacings to evaluate the masses of strange and charm baryons. The strange and charm quarks are added as valence quarks.

For heavy quarks, the Compton wavelength of the associated heavy-light meson is comparable to presently attainable lattice spacings, which means that cutoff effects may be large. The charm quark mass is at the upper limit of the range of masses that can be directly simulated at present. In order to obtain values for the masses that can be compared to experiment, it is important to assess the size of lattice artifacts. A first study of cutoff effects was carried out for light and strange baryons in Refs. [1,2]. In this work, we extend the study by including a finer lattice spacing and calculate the masses of charm baryons in addition to the masses of strange baryons. Having three lattice spacings, the continuum extrapolation can be better assessed.
In this work, we compare our results in the strange baryon sector with recent results obtained with Clover-improved Wilson fermions with different levels of smearing. The PACS-CS [3] and BMW [4] collaborations evaluated the octet spectrum using two degenerate flavors of light quarks and a strange quark with mass tuned to its physical value. The PACS-CS has also computed the decuplet baryon masses. In addition, we compare our data with the LHPC, which computed the octet and decuplet spectrum using a hybrid action with domain-wall valence fermions on KogutSusskind sea quarks [5].

Besides the strange baryons, we also study the ground state spectrum of charm baryons with spin $J=1 / 2^{+}$and spin $J=3 / 2^{+}$. Experimental searches of charm hadrons have received significant attention, mainly due to the experimental observation for candidates of the doubly charmed baryons $\Xi_{c c}^{+}(3520)$ and $\Xi_{c c}^{++}(3460)$ by the SELEX Collaboration [6-8]. The $60 \mathrm{MeV}$ mass difference between the singly and doubly charged states is difficult to understand, since it is an order of magnitude larger compared to what is expected. No evidence was found for these states by the BABAR experiment [9] and FOCUS Collaboration [10]. The Belle Collaboration [11] finds $\Xi$ states lower in mass that can be candidates of excited states of $\Xi_{c}$, but no doubly charmed $\Xi$ baryons. Additional experiments are planned at the new Beijing Spectrometer (BES-III) and at the antiProton ANnihilation at DArmstadt (PANDA) experiment at GSI, that can shed light on these charm baryon states. Several lattice QCD studies have been carried out to study charm baryons. We will compare the results of the current work with recent lattice QCD results, all computed in a hybrid action approach where the charm 
valence quark was introduced on gauge configurations produced with staggered sea fermions by the MILC Collaboration [12-14].

As in the case of the other lattice QCD studies of heavy baryons, in this work we use a mixed action approach. For the strange and charm sector, we use an OsterwalderSeiler valence quark, following the approach employed in the study of the pseudoscalar meson decay constants $[15,16]$. The bare strange and charm valence quark mass is tuned by requiring that the physical values of the masses of the kaon and $D$ meson are reproduced after the lattice results are extrapolated at the physical value of the pion mass. The ETMC $N_{f}=2$ configurations $[17,18]$ analyzed in this work correspond to pion masses in the range of 260 to $450 \mathrm{MeV}$ and three values of the lattice spacing corresponding to $\beta=3.9,4.05$ and 4.2 , with $r_{0} / a=5.22(2), 6.61(3)$ and 8.31(5), respectively. The Sommer parameter $r_{0}$ is determined from the force between two static quarks, the continuum value of which is determined to be $0.462(5) \mathrm{fm}$. At $\beta=4.2$ we use two ensembles, one corresponding to the lowest value of the pion mass considered in this work and one corresponding to the upper pion mass range. We find that the baryon masses, in general, show a very weak dependence on the lattice spacing and are fully compatible with an $\mathcal{O}\left(a^{2}\right)$ behaviour with an almost vanishing coefficient of the $a^{2}$ term. This justifies neglecting the $\mathcal{O}\left(a^{2}\right)$ term in extrapolating results to the continuum limit.

An important issue raised by the twisted mass fermion formulation is isospin symmetry breaking. This symmetry, although exact in the continuum limit, is broken at a nonvanishing lattice spacing to $\mathcal{O}\left(a^{2}\right)$. There are, however, theoretical arguments [19] and numerical evidences $[20,21]$ that these isospin breaking effects are only sizable for the neutral pseudoscalar mass, whereas for other quantities studied so far by ETMC they are compatible with zero. In this paper, we demonstrate that in the baryon sector also, these isospin breaking effects are, in general, small or even compatible with zero. Small isospin breaking effects decrease as the lattice spacing decreases, and they vanish at the continuum limit. This corroborates our previous findings [1,2]. The isospin breaking effects are relevant not only for neutral pions but also for other particles, e.g., the kaons. However, since the mass of the kaon is higher, the relative splitting (between $K^{0}$ and $K^{+}$) is less drastic.

The paper is organized as follows: The details of our lattice formulation, namely those concerning the twisted mass action, the parameters of the simulations, the interpolating fields used, and the tuning of the strange and charm quark masses are given in Sec. II. Section III contains the numerical results of the baryon masses computed for different lattice volumes, lattice spacings and bare quark masses. Lattice artifacts, including finite volume and discretization errors, and continuum extrapolation are also discussed in Sec. III, with special emphasis on the
$\mathcal{O}\left(a^{2}\right)$ isospin breaking effects inherent to the twisted mass formulation of lattice QCD. The chiral extrapolations are analyzed in Sec. IV. Section V contains a comparison with other existing calculations. Our conclusions are finally drawn in Sec. VI.

\section{LATTICE FORMULATION}

\section{A. The lattice action}

For the gauge fields, we use the tree-level Symanzik improved gauge action [22], which includes, besides the plaquette term $U_{x, \mu, \nu}^{1 \times 1}$, rectangular $(1 \times 2)$ Wilson loops $U_{x, \mu, \nu}^{1 \times 2}$ :

$$
\begin{aligned}
S_{g}= & \frac{\beta}{3} \sum_{x}\left(b_{0} \sum_{\substack{\mu, \nu=1 \\
1 \leq \mu<\nu}}^{4}\left\{1-\operatorname{Re} \operatorname{Tr}\left(U_{x, \mu, \nu}^{1 \times 1}\right)\right\}\right. \\
& \left.+b_{1} \sum_{\substack{\mu, \nu=1 \\
\mu \neq \nu}}^{4}\left\{1-\operatorname{Re} \operatorname{Tr}\left(U_{x, \mu, \nu}^{1 \times 2}\right)\right\}\right),
\end{aligned}
$$

with $b_{1}=-1 / 12$ and the (proper) normalization condition $b_{0}=1-8 b_{1}$. Note that at $b_{1}=0$, this action becomes the usual Wilson plaquette gauge action.

The fermionic action for two degenerate flavors of quarks in twisted mass QCD is given by

$$
S_{F}=a^{4} \sum_{x} \bar{\chi}(x)\left(D_{W}[U]+m_{0}+i \mu \gamma_{5} \tau^{3}\right) \chi(x),
$$

with $\tau^{3}$ the Pauli matrix acting in the isospin space, $\mu$ the bare twisted mass, and the massless Wilson-Dirac operator given by

$$
D_{W}[U]=\frac{1}{2} \gamma_{\mu}\left(\nabla_{\mu}+\nabla_{\mu}^{*}\right)-\frac{a r}{2} \nabla_{\mu} \nabla_{\mu}^{*}
$$

where

$\nabla_{\mu} \psi(x)=\frac{1}{a}\left[U_{\mu}^{\dagger}(x) \psi(x+a \hat{\mu})-\psi(x)\right]$ and

$\nabla_{\mu}^{*} \psi(x)=-\frac{1}{a}\left[U_{\mu}(x-a \hat{\mu}) \psi(x-a \hat{\mu})-\psi(x)\right]$.

Maximally twisted Wilson quarks are obtained by setting the untwisted quark mass $m_{0}$ to its critical value $m_{\mathrm{cr}}$, while the twisted quark mass parameter $\mu$ is kept nonvanishing in order to work away from the chiral limit. In Eq. (2), the quark fields $\chi$ are in the so-called "twisted basis." The "physical basis" is obtained for maximal twist by the simple transformation

$$
\begin{aligned}
& \psi(x)=\exp \left(\frac{i \pi}{4} \gamma_{5} \tau^{3}\right) \chi(x), \\
& \bar{\psi}(x)=\bar{\chi}(x) \exp \left(\frac{i \pi}{4} \gamma_{5} \tau^{3}\right)
\end{aligned}
$$

In terms of the physical fields, the action is given by 


$$
\begin{aligned}
S_{F}^{\psi}= & a^{4} \sum_{x} \bar{\psi}(x)\left(\frac{1}{2} \gamma_{\mu}\left[\nabla_{\mu}+\nabla_{\mu}^{*}\right]\right. \\
& \left.-i \gamma_{5} \tau^{3}\left(-\frac{a r}{2} \nabla_{\mu} \nabla_{\mu}^{*}+m_{\mathrm{cr}}\right)+\mu\right) \psi(x) .
\end{aligned}
$$

In this paper, unless otherwise stated, the quark fields will be understood as physical fields, $\psi$, in particular when we define the baryonic interpolating fields.

A crucial advantage of the twisted mass formulation is the fact that, by tuning the bare untwisted quark mass $m_{0}$ to its critical value $m_{\mathrm{cr}}$, all physical observables are automatically $\mathcal{O}(a)$-improved. In practice, we implement maximal twist of Wilson quarks by tuning to zero the bare untwisted current quark mass, commonly called the PCAC mass, $m_{\mathrm{PCAC}}$ [23], which is proportional to $m_{0}-m_{\mathrm{cr}}$ up to $\mathcal{O}(a)$ corrections. The value of $m_{\mathrm{cr}}$ is determined at each $\beta$ value at the lowest twisted mass used in our simulations, a procedure that preserves $\mathcal{O}(a)$ improvement and keeps $\mathcal{O}\left(a^{2}\right)$ small $[18,24]$. The twisted mass fermionic action breaks parity and isospin at nonvanishing lattice spacing, as is apparent from the form of the Wilson term in Eq. (6). In particular, the isospin breaking in physical observables is a cutoff effect of $\mathcal{O}\left(a^{2}\right)$ [25]. To simulate the strange quark in the valence sector, several choices are possible.

The strange and charm quarks are added as OsterwalderSeiler valence quarks, and their action reads

$$
\begin{aligned}
S_{\text {heavy }}^{\text {OS }}= & a^{4} \sum_{x} \sum_{h=s}^{c} \bar{\chi}_{h}(x)\left(\frac{\gamma_{\mu}}{2}\left(\nabla_{\mu}+\nabla_{\mu}^{*}\right)-\frac{a}{2} \nabla_{\mu}^{*} \nabla_{\mu}\right. \\
& \left.+M_{\text {cr }}+i \gamma_{5} \mu_{h}\right) \chi_{h}(x),
\end{aligned}
$$

where $\mu_{s}$ and $\mu_{c}$ are the strange and charm valence quark masses. This is naturally realized in the twisted mass approach by introducing two additional doublets of strange and charm quarks and keeping only the positive diagonal component of $\tau_{3}$. The $m_{0}$ value is taken to be equal to the critical mass determined in the light sector, thus guaranteeing the $O(a)$ improvement in any observable. The reader interested in the advantage of this mixed action in the mesonic sector is referred to Refs. [15,16,26-28].

\section{B. Simulation details}

The input parameters of the calculation, namely $\beta, L / a$ and $a \mu$, are summarized in Table I. The corresponding

TABLE I. Input parameters $(\beta, L, \mu)$ of our lattice simulations and corresponding lattice spacing $(a)$ and pion mass $\left(m_{\pi}\right)$. The statistics refer to the number of configurations used in the calculation of the masses of the strange and charm baryons. The first entry gives the number

\begin{tabular}{|c|c|c|c|c|c|}
\hline \multicolumn{6}{|c|}{$\beta=4.2, a=0.056(1) \mathrm{fm} r_{0} / a=8.31(5)$} \\
\hline $32^{3} \times 64, L=1.8 \mathrm{fm}$ & $\begin{array}{c}a \mu_{\text {sea }} \\
\text { statistics } \\
m_{\pi}(\mathrm{GeV}) \\
m_{\pi} L\end{array}$ & $\begin{array}{c}0.0065 \\
240,76 \\
0.4698(18) \\
4.24\end{array}$ & & & \\
\hline $48^{3} \times 92, L=2.7 \mathrm{fm}$ & $\begin{array}{c}a \mu_{\text {sea }} \\
\text { statistics } \\
m_{\pi}(\mathrm{GeV}) \\
m_{\pi} L\end{array}$ & $\begin{array}{c}0.0020 \\
458,456 \\
0.262(1) \\
3.55\end{array}$ & & & \\
\hline \multicolumn{6}{|c|}{$\beta=4.05, a=0.070(1) \mathrm{fm}, r_{0} / a=6.61(3)$} \\
\hline $32^{3} \times 64, L=2.13 \mathrm{fm}$ & $\begin{array}{c}a \mu_{\text {sea }} \\
\text { statistics } \\
m_{\pi}(\mathrm{GeV}) \\
m_{\pi} L\end{array}$ & $\begin{array}{c}0.0030 \\
144,144 \\
0.2925(18) \\
3.31\end{array}$ & $\begin{array}{c}0.0060 \\
194,193 \\
0.4035(18) \\
4.57\end{array}$ & $\begin{array}{c}0.0080 \\
201,201 \\
0.4653(15) \\
5.27\end{array}$ & \\
\hline \multicolumn{6}{|c|}{$\beta=3.9, a=0.089(1) \mathrm{fm}, r_{0} / a=5.22(2)$} \\
\hline $24^{3} \times 48, L=2.05 \mathrm{fm}$ & $\begin{array}{c}a \mu_{\mathrm{sea}} \\
\text { statistics } \\
m_{\pi}(\mathrm{GeV}) \\
m_{\pi} L\end{array}$ & $\begin{array}{c}0.0040 \\
4112,310 \\
0.3032(16) \\
3.25\end{array}$ & $\begin{array}{c}0.0064 \\
545,278 \\
0.3770(9) \\
4.05\end{array}$ & $\begin{array}{c}0.0085 \\
1817,369 \\
0.4319(12) \\
4.63\end{array}$ & $\begin{array}{c}0.010 \\
477,475 \\
0.4675(12) \\
5.03\end{array}$ \\
\hline $32^{3} \times 64, L=2.74 \mathrm{fm}$ & $\begin{array}{c}a \mu_{\text {sea }} \\
\text { statistics } \\
m_{\pi}(\mathrm{GeV}) \\
m_{\pi} L\end{array}$ & $\begin{array}{c}0.0030 \\
659, \cdots \\
0.2600(9) \\
3.74\end{array}$ & $\begin{array}{c}0.0040 \\
242, \cdots \\
0.2978(6) \\
4.28\end{array}$ & & \\
\hline
\end{tabular}
used for the tuned value of the strange quark; the second, for the tuned value of the charm. An entry of "..." indicates that no mass was computed. The lattice spacing was determined using the nucleon mass [29]. 
lattice spacing $a$ and the pion mass values, spanning a mass range from 260 to $450 \mathrm{MeV}$, are taken from Ref. [29]. At $m_{\pi} \approx 300 \mathrm{MeV}$ we have simulations for lattices of spatial sizes $L=2.1 \mathrm{fm}$ and $L=2.7 \mathrm{fm}$ at $\beta=3.9$, allowing us to investigate finite size effects. Finite lattice-spacing effects are investigated using three sets of results at $\beta=3.9$, $\beta=4.05$, and $\beta=4.2$. These sets of gauge ensembles allow us to estimate all the systematic errors in order to have reliable predictions for the baryon spectrum.

\section{Tuning of the bare strange and charm quark masses}

The dependence of the pseudoscalar meson mass on the valence and sea quarks can be written as a polynomial of the form [15]

$$
\begin{aligned}
& a^{2} M_{\mathrm{PS}}^{2}\left(a \mu_{\text {sea }}, a \mu_{1}, \mu_{2}\right) \\
& =B_{0}\left(a \mu_{1}+a \mu_{2}\right)\left[1+a_{V} \xi_{12}+a_{\text {sea }} \xi_{\text {sea }}+a_{V V} \xi_{12}^{2}\right. \\
& \left.\quad+a_{\text {sea }}^{\prime} \xi_{\text {sea }}^{2}+a_{V \text {,sea }} \xi_{12} \xi_{\text {sea }}+a_{V D} \xi_{D 12}^{2}\right],
\end{aligned}
$$

where $\mu_{\text {sea }}$ is the sea quark mass, $a \mu_{1}$ and $a \mu_{2}$ are the valence quark masses, $\xi_{i}=B_{0} a \mu_{i} /(4 \pi f)^{2}, \xi_{i j}=2 B_{0}\left(a \mu_{i}+a \mu_{j}\right) /$ $(4 \pi f)^{2}$, and $\xi_{D i j}=B_{0}\left(a \mu_{i}-a \mu_{j}\right) /(4 \pi f)^{2}$. For the $\beta=3.9$ ensembles, we consider in total 164 pseudoscalar meson masses using all possible combinations of sea and valence quark masses. Namely, we consider 150 combinations obtained from $a \mu_{\text {sea }}$ and $a \mu_{1}$ independently taking the values

$$
\left\{a \mu_{\text {sea }}, a \mu_{1}\right\}=\left\{\begin{array}{lllll}
0.0040 & 0.0064 & 0.0085 & 0.0100 & 0.0150
\end{array}\right\},
$$

whereas $a \mu_{2}$ takes the values

$$
\begin{aligned}
a \mu_{2}= & \left\{\begin{array}{lllll}
0.0040 & 0.0064 & 0.0085 & 0.0100 & 0.0150 \\
& 0.0220 & 0.0270 & 0.0320
\end{array}\right\} .
\end{aligned}
$$

We have an additional 12 combinations coming from

$$
\begin{aligned}
a \mu_{\text {sea }} & =\mu_{1}=\left\{\begin{array}{llll}
0.0040 & 0.0064 & 0.0085 & 0.0100
\end{array}\right\}, \\
\mu_{2} & =\left\{\begin{array}{lll}
0.24 & 0.27 & 0.30
\end{array}\right\}
\end{aligned}
$$

plus two extra combinations from

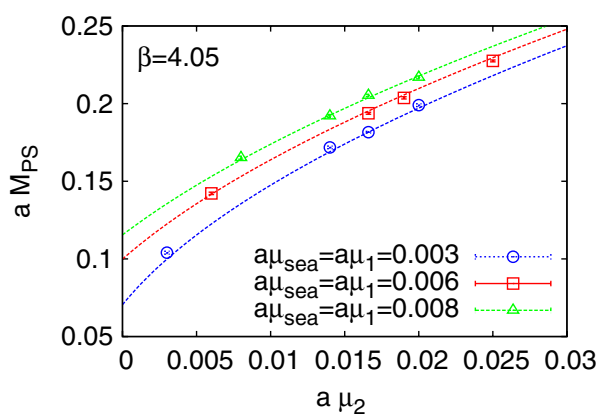

$$
a \mu_{\text {sea }}=a \mu_{1}=0.0040, \quad a \mu_{2}=\left\{\begin{array}{ll}
0.0217 & 0.25
\end{array}\right\} .
$$

For the tuning at $\beta=4.05$, we use the following 20 combinations:

$$
\begin{aligned}
& a \mu_{\mathrm{sea}}=a \mu_{1}=0.0030 \text {, } \\
& a \mu_{2}=\left\{\begin{array}{lllll}
0.0030 & 0.014 & 0.0166 & 0.020 & 0.17
\end{array}\right. \\
& \begin{array}{lll}
0.20 & 0.23 & 0.26
\end{array} \\
& a \mu_{\mathrm{sea}}=a \mu_{1}=0.0060 \text {, } \\
& a \mu_{2}=\left\{\begin{array}{llll}
0.0060 & 0.0166 & 0.019 & 0.025
\end{array}\right\} ; \\
& a \mu_{\mathrm{sea}}=a \mu_{1}=0.0080 \text {, } \\
& a \mu_{2}=\left\{\begin{array}{lllll}
0.0080 & 0.014 & 0.0166 & 0.020 & 0.17
\end{array}\right. \\
& \begin{array}{lll}
0.20 & 0.23 & 0.26
\end{array} \text {. }
\end{aligned}
$$

For the tuning at $\beta=4.2$, we consider ten pseudoscalar meson masses:

$$
\begin{aligned}
a \mu_{\text {sea }} & =a \mu_{1}=0.0065, \\
a \mu_{2} & =\left\{\begin{array}{lllll}
0.0065 & 0.14 & 0.16 & 0.185 & 0.21
\end{array}\right\} ; \\
a \mu_{\text {sea }} & =a \mu_{1}=0.0020, \\
a \mu_{2} & =\left\{\begin{array}{lllll}
0.0020 & 0.012 & 0.015 & 0.136 & 0.17
\end{array}\right\} .
\end{aligned}
$$

In Figs. 1 and 2, we show representative fits to the pseudoscalar masses in the range of the kaon and $D$-meson masses using the expression given in Eq. (8). The values of the strange and charm quark masses are varied until the resulting kaon and $D$-meson masses are matched to their physical values. The resulting fit parameters are listed in Table II. We note that for $\beta=3.9$, two fitting ranges are used-one range spanning the strange quark mass, and one the charm quark mass. For $\beta=4.05$ and $\beta=4.2$ we fit all data together, since we do not have enough mass combinations in order to apply Eq. (8). If one does the same for $\beta=3.9$, then the tuned value for the strange quark mass is $a \mu_{s}=0.0216(7)$, compatible with the value of $a \mu_{s}=0.0217(5)$ if we restrict the fit to the strange region. In addition, at each $\beta$ value we can restrict the fit in the charm region using the ansatz

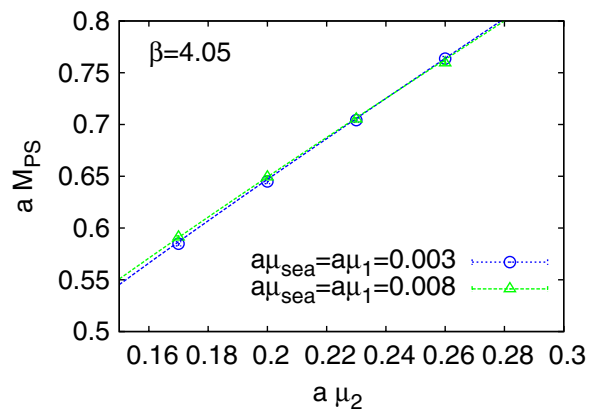

FIG. 1 (color online). Pseudoscalar meson masses for $\beta=4.05$ as a function of the heavy valence quark mass $a \mu_{2}$ in the relevant mass range for the strange quark (left) and charm quark (right). In all the examples shown, the sea quark mass $a \mu_{\text {sea }}$ is set equal to the light valence quark mass $a \mu_{1}$. 

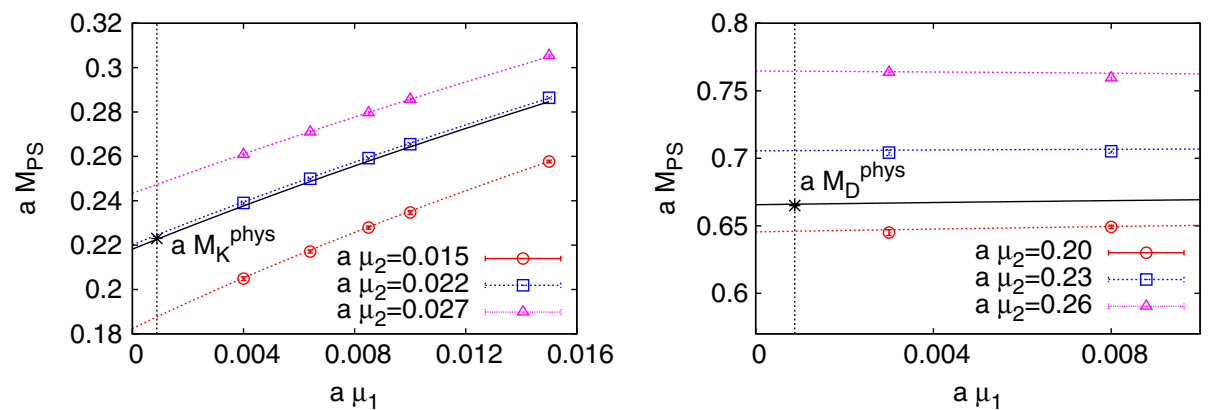

FIG. 2 (color online). The dependence of the pseudoscalar masses on the strange quark mass at $\beta=3.9$ (left) and the charm quark mass at $\beta=4.05$ (right). In each plot, the solid line shows the variation of the pseudoscalar mass at the tuned strange (left) and charm (right) quark masses. The dashed vertical line corresponds to the value of $a \mu_{1}$ at which the physical pion mass is recovered. The asterisk denotes the physical value of the kaon or $D$-meson mass in lattice units. The lattice spacing is determined from the nucleon mass.

$$
m_{B}=m_{B}^{0}+b \mu_{h}+c / \mu_{h}
$$

The tuned charm quark value is found to be compatible with the one extracted using Eq. (8). This procedure can be carried out using the lattice spacing determined either from the nucleon mass or from $f_{\pi}$. The difference in the tuned masses reflects the systematic error in setting the scale.

The tuned values of the strange and charm quark masses $m_{s}$ and $m_{c}$ obtained at the physical pion are given in Table III. In a previous paper, the ETMC computed pseudoscalar meson masses for a number of sea and valence quark masses using the $\beta=3.9$ gauge configurations. By matching the experimental value of the mass ratio of the kaon to the pion, $m_{K} / m_{\pi}$, the bare strange quark mass was determined [15]. Depending on the polynomial fit used, the values for $a m_{s}$ at $\beta=3.9$ varied from 0.0243(5) to 0.0218 (10). Thus, our value of $a \mu_{s}=0.0216(7)$ from matching the physical value of the kaon mass in combination with the lattice spacing determined from the nucleon mass is compatible with the value determined in Ref. [15]. Such an agreement is satisfactory and shows that the two procedures lead to the same determination within the uncertainties associated with the extrapolation. The systematic error introduced from the way the lattice scale is fixed can be assessed by comparing the tuned values extracted using the lattice spacing determined from the nucleon mass and from

TABLE II. The values of the fit parameters.

\begin{tabular}{lcccc}
\hline \hline & $\beta=3.9$ (Strange quark) & $\beta=3.9$ (Charm quark) & $\beta=4.05$ & $\beta=4.2$ \\
\hline$B_{0}$ & $2.252(5)$ & $2.38(6)$ & $1.652(5)$ & $1.295(5)$ \\
$f$ & $0.077(2)$ & $0.112(2)$ & $0.093(4)$ & $0.069(4)$ \\
$a_{V}$ & $-0.45(2)$ & $0.3(1)$ & $0.85(5)$ & $0.56(5)$ \\
$a_{\text {sea }}$ & 0.0 & 0.0 & 0.0 & 0.0 \\
$a_{V V}$ & $3.0(1)$ & $1.8(5)$ & $-4.0(3)$ & $2.6(2)$ \\
$a_{V \text { sea }}^{\prime}$ & 0.0 & 0.0 & 0.0 & 0.0 \\
$a_{\text {sea }}^{\prime}$ & 0.0 & 0.0 & 0.0 & 0.0 \\
$a_{V D}$ & $-2.25(3)$ & $-1.4(6)$ & $4.94(5)$ & $-1.8(3)$ \\
$\chi^{2} /$ d.o.f. & 0.51 & 1.33 & 4.52 & 4.40 \\
\hline \hline
\end{tabular}

TABLE III. The strange and charm quark masses at each value of $\beta$, tuned using the kaon and $D$-meson masses; the lattice spacing determined from the nucleon masses is given in lattice units in the second and third columns for strange and charm quarks, respectively. The tuned strange and charm quark masses in $\mathrm{GeV}$ are given in the fourth and fifth columns. In the sixth and seventh columns, we give the corresponding masses in $\mathrm{GeV}$ using the lattice determined from $f_{\pi}$.

\begin{tabular}{lllllll}
\hline \hline$\beta$ & $a \mu_{s}$ & $a \mu_{c}$ & $\mu_{s}(\mathrm{GeV})$ & $\mu_{c}(\mathrm{GeV})$ & $\mu_{s}^{f_{\pi}}(\mathrm{GeV})$ & $\mu_{c}^{f_{\pi}}(\mathrm{GeV})$ \\
\hline 3.9 & $0.0216(7)$ & $0.27(3)$ & $0.0478(16)$ & $0.598(66)$ & $0.0431(17)$ & $0.64(12)$ \\
4.05 & $0.0178(5)$ & $0.21(1)$ & $0.0501(14)$ & $0.591(28)$ & $0.0451(12)$ & $0.556(31)$ \\
4.2 & $0.014(1)$ & $0.17(2)$ & $0.0493(35)$ & $0.598(70)$ & $0.0464(15)$ & $0.575(38)$ \\
\hline \hline
\end{tabular}




$$
\begin{aligned}
& s=0 \\
& s=-1 \\
& s=-2
\end{aligned}
$$

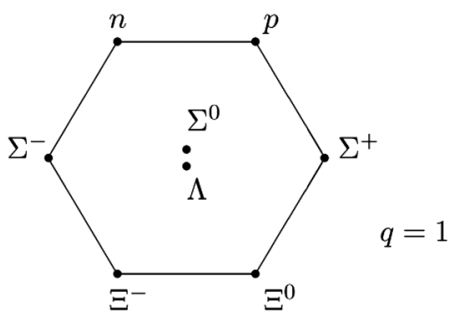

$$
q=-1 \quad q=0
$$

FIG. 3. The low-lying baryons belonging to the octet representation, labeled by value of $I_{3}$ and hypercharge.

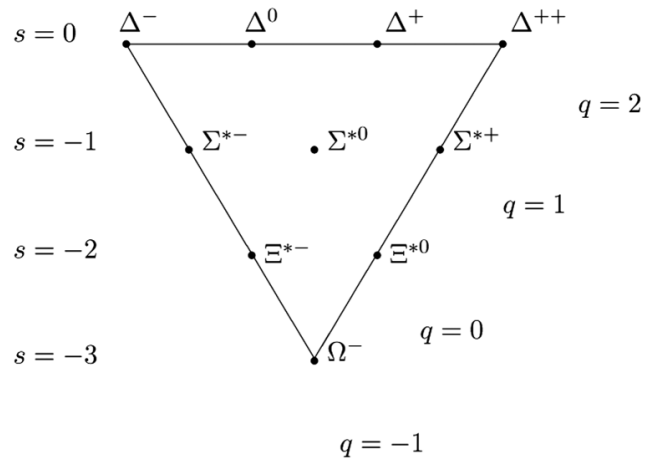

FIG. 4. The low-lying baryons belonging to the decuplet representation, labeled by value of $I_{3}$ and hypercharge.

the pion decay constant $f_{\pi}$. The values of the lattice spacing determined using $f_{\pi}$, taken from Ref. [30], are $a_{\beta=3.9}=0.0801(14) \mathrm{fm}, a_{\beta=4.05}=0.0638(10) \mathrm{fm}$, and $a_{\beta=4.2}=0.05142(38) \mathrm{fm}$. In Table III, we give the tuned values for the charm and strange quark masses expressed in physical units. As can be seen, the values for the charm quark masses are in agreement, whereas for strange quark masses the differences are within about two standard deviations.

\section{Interpolating fields}

The low-lying baryons belonging to the octet and decuplet representations of $S U(3)$ are given in Figs. 3 and 4, respectively. They are classified by giving the isospin $I$, the third component of the isospin $I_{3}$, the strangeness $S$, the spin, and the parity. In order to extract their masses in lattice QCD, we evaluate two-point correlators. We use interpolating fields to create states from the vacuum that have the correct quantum numbers and reduce to the quark model wave functions in the nonrelativistic limit. The interpolating fields used in this work are collected in Tables IV [31,32] and V [31,33] for the octet and decuplet, respectively.

Charm baryons with no strange quarks are obtained from the interpolating fields of strange baryons by replacing the strange with the charm quark. There are additional charm baryons containing strange quarks, giving a 20-plet of spin-1/2 and a 20-plet of spin-3/2. In most of this work, we do not consider the particles that contain both strange and charm quarks. For the lattice with the smallest lattice spacing and at the smallest pion mass, we also consider the spin- $1 / 2 \Xi_{c}, \Xi_{c}^{\prime}, \Omega_{c}$ and $\Omega_{c c}$ baryons and the spin-3/2 $\Xi_{c}^{*}, \Omega_{c}^{*}$ and $\Omega_{c c}^{*}$ baryons. The interpolating fields for these baryons are given in Table VI.

Local interpolating fields are not optimal for suppressing excited-state contributions. We instead apply Gaussian smearing to each quark field, $q(\mathbf{x}, t): q^{\text {smear }}(\mathbf{x}, t)=$ $\sum_{\mathbf{y}} F(\mathbf{x}, \mathbf{y} ; U(t)) q(\mathbf{y}, t)$, using the gauge-invariant smearing function

$$
F(\mathbf{x}, \mathbf{y} ; U(t))=(1+\alpha H)^{n}(\mathbf{x}, \mathbf{y} ; U(t)),
$$

constructed from the hopping matrix,

$H(\mathbf{x}, \mathbf{y} ; U(t))=\sum_{i=1}^{3}\left(U_{i}(\mathbf{x}, t) \delta_{\mathbf{x}, \mathbf{y}-i}+U_{i}^{\dagger}(\mathbf{x}-i, t) \delta_{\mathbf{x}, \mathbf{y}+i}\right)$.

Furthermore, we apply APE smearing to the spatial links that enter the hopping matrix. The parameters of the Gaussian and APE smearing are the same as those used in our previous work devoted to the nucleon and $\Delta$ masses [1].

\section{E. Two-point correlators}

To extract masses in the rest frame, we consider twopoint correlators, defined by

TABLE IV. Interpolating fields and quantum numbers for baryons in the octet representation.

\begin{tabular}{lcccc}
\hline \hline Strangeness & Baryon & Interpolating field & $I$ & $I_{z}$ \\
\hline \multirow{2}{*}{$S=0$} & $p$ & $\chi^{p}=\epsilon_{a b c}\left(u_{a}^{T} C \gamma_{5} d_{b}\right) u_{c}$ & $1 / 2$ & $+1 / 2$ \\
& $n$ & $\chi^{n}=\epsilon_{a b c}\left(d_{a}^{T} C \gamma_{5} u_{b}\right) d_{c}$ & $1 / 2$ & $-1 / 2$ \\
& $\Lambda$ & $\chi^{\Lambda^{8}}=\frac{1}{\sqrt{6}} \epsilon_{a b c}\left\{2\left(u_{a}^{T} C \gamma_{5} d_{b}\right) s_{c}+\left(u_{a}^{T} C \gamma_{5} s_{b}\right) d_{c}-\left(d_{a}^{T} C \gamma_{5} s_{b}\right) u_{c}\right\}$ & 0 & 0 \\
$S=1$ & $\Sigma^{+}$ & $\chi^{\Sigma^{+}}=\epsilon_{a b c}\left(u_{a}^{T} C \gamma_{5} s_{b}\right) u_{c}$ & 1 & +1 \\
& $\Sigma^{0}$ & $\Sigma^{\Sigma^{0}}=\frac{1}{\sqrt{2}} \epsilon_{a b c}\left\{\left(u_{a}^{T} C \gamma_{5} s_{b}\right) d_{c}+\left(d_{a}^{T} C \gamma_{5} s_{b}\right) u_{c}\right\}$ & 1 & +0 \\
& $\Sigma^{-}$ & $\chi^{\Sigma^{-}}=\epsilon_{a b c}\left(d_{a}^{T} C \gamma_{5} s_{b}\right) d_{c}$ & 1 & -1 \\
$S=2$ & $\Xi^{0}$ & $\chi^{\Xi^{0}}=\epsilon_{a b c}\left(s_{a}^{T} C \gamma_{5} u_{b}\right) s_{c}$ & $1 / 2$ & $+1 / 2$ \\
& $\Xi^{-}$ & $\chi^{\Xi^{-}}=\epsilon_{a b c}\left(s_{a}^{T} C \gamma_{5} d_{b}\right) s_{c}$ & $1 / 2$ & $-1 / 2$ \\
\hline \hline
\end{tabular}


TABLE V. Interpolating fields and quantum numbers for baryons in the decuplet representation.

\begin{tabular}{|c|c|c|c|c|}
\hline Strangeness & Baryon & Interpolating field & $I$ & $I_{z}$ \\
\hline \multirow[t]{2}{*}{$S=0$} & $\begin{array}{c}\Delta^{++} \\
\Delta^{+}\end{array}$ & $\begin{array}{c}\chi_{\mu}^{\Delta^{++}}=\epsilon_{a b c}\left(u_{a}^{T} C \gamma_{\mu} u_{b}\right) u_{c} \\
\chi_{\mu}^{\Delta^{+}}=\frac{1}{\sqrt{3}} \epsilon_{a b c}\left\{2\left(u_{a}^{T} C \gamma_{\mu} d_{b}\right) u_{c}+\left(u_{a}^{T} C \gamma_{\mu} u_{b}\right) d_{c}\right\}\end{array}$ & $\begin{array}{l}3 / 2 \\
3 / 2\end{array}$ & $\begin{array}{l}+3 / 2 \\
+1 / 2\end{array}$ \\
\hline & $\begin{array}{l}\Delta^{0} \\
\Delta^{-}\end{array}$ & $\begin{array}{c}\chi_{\mu}^{\Delta^{0}}=\frac{1}{\sqrt{3}} \epsilon_{a b c}\left\{2\left(d_{a}^{T} C \gamma_{\mu} u_{b}\right) d_{c}+\left(d_{a}^{T} C \gamma_{\mu} d_{b}\right) u_{c}\right\} \\
\chi_{\mu}^{\Delta^{-}}=\epsilon_{a b c}\left(d_{a}^{T} C \gamma_{\mu} d_{b}\right) d_{c}\end{array}$ & $\begin{array}{l}3 / 2 \\
3 / 2\end{array}$ & $\begin{array}{l}-1 / 2 \\
-3 / 2\end{array}$ \\
\hline \multirow[t]{3}{*}{$S=1$} & $\Sigma^{*+}$ & $\chi_{\mu}^{\sum^{*+}}=\sqrt{\frac{2}{3}} \epsilon^{a b c}\left\{\left(u^{T a} C \gamma_{\mu} u^{b}\right) s^{c}+2\left(u^{T a} C \gamma_{\mu} s^{b}\right) u^{c}\right\}$ & 1 & +1 \\
\hline & $\Sigma^{* 0}$ & $\chi_{\mu}^{\Sigma^{* 0}}=\sqrt{\frac{2}{3}} \epsilon^{a b c}\left\{\left(u^{T a} C \gamma_{\mu} d^{b}\right) s^{c}+\left(d^{T a} C \gamma_{\mu} s^{b}\right) u^{c}+\left(s^{T a} C \gamma_{\mu} u^{b}\right) d^{c}\right\}$ & 1 & +0 \\
\hline & $\Sigma^{*-}$ & $\chi_{\mu}^{\Sigma^{*-}}=\sqrt{\frac{2}{3}} \epsilon^{a b c}\left\{\left(d^{T a} C \gamma_{\mu} d^{b}\right) s^{c}+2\left(d^{T a} C \gamma_{\mu} s^{b}\right) d^{c}\right\}$ & 1 & -1 \\
\hline$S=2$ & $\Xi^{* 0}$ & $\begin{aligned} \chi \bar{\Xi}^{* 0} & =\epsilon_{a b c}\left(s_{a}^{T} C \gamma_{\mu} u_{b}\right) s_{c} \\
\chi_{\mu}^{*-} & =\epsilon_{a b c}\left(s_{a}^{T} C \gamma_{\mu} d_{b}\right) s_{c}\end{aligned}$ & $\begin{array}{l}1 / 2 \\
1 / 2\end{array}$ & $\begin{array}{l}+1 / 2 \\
-1 / 2\end{array}$ \\
\hline$S=3$ & $\Omega^{-}$ & $\chi_{\mu}^{\Omega^{-}}=\epsilon_{a b c}\left(s_{a}^{T} C \gamma_{\mu} s_{b}\right) s_{c}$ & 0 & +0 \\
\hline
\end{tabular}

TABLE VI. Interpolating fields for the spin-1/2 $\Xi_{c}, \Xi_{c}^{\prime}, \Omega_{c}$ and $\Omega_{c c}$ baryons, and the spin-3/2 $\Xi_{c}^{*}, \Omega_{c}^{*}$ and $\Omega_{c c}^{*}$ baryons.

\begin{tabular}{|c|c|}
\hline$J=1 / 2$ & $J=3 / 2$ \\
\hline$\chi^{\Xi_{c}}=\frac{1}{\sqrt{6}} \epsilon_{a b c}\left\{2\left(s_{a}^{T} C \gamma_{5} d_{b}\right) c_{c}+\left(s_{a}^{T} C \gamma_{5} c_{b}\right) d_{c}-\left(d_{a}^{T} C \gamma_{5} c_{b}\right) s_{c}\right\}$ & $\chi_{\mu}^{\Xi_{c}^{*}}=\sqrt{\frac{2}{3}} \epsilon^{a b c}\left\{\left(s^{T a} C \gamma_{\mu} d^{b}\right) c^{c}+\left(d^{T a} C \gamma_{\mu} c^{b}\right) s^{c}+\left(c^{T a} C \gamma_{\mu} s^{b}\right) d^{c}\right\}$ \\
\hline $\begin{array}{l}\chi^{\Xi_{c}^{\prime}}=\frac{1}{\sqrt{2}} \epsilon_{a b c}\left\{\left(s_{a}^{T} C \gamma_{5} c_{b}\right) d_{c}+\left(d_{a}^{T} C \gamma_{5} c_{b}\right) s_{c}\right\} \\
\chi^{\Omega_{c}}=\epsilon_{a b c}\left(s_{a}^{T} C \gamma_{5} c_{b}\right) s_{c} \\
\chi^{\Omega_{c c}}=\epsilon_{a b c}\left(c_{a}^{T} C \gamma_{5} s_{b}\right) c_{c}\end{array}$ & $\begin{array}{c}\chi_{\mu}^{\Omega_{c}^{*}}=\sqrt{\frac{2}{3}} \epsilon^{a b c}\left\{\left(s^{T a} C \gamma_{\mu} s^{b}\right) c^{c}+2\left(s^{T a} C \gamma_{\mu} c^{b}\right) s^{c}\right\} \\
\chi_{\mu}^{\Omega_{c c}^{*}}=\epsilon_{a b c}\left(c_{a}^{T} C \gamma_{\mu} s_{b}\right) c_{c}\end{array}$ \\
\hline
\end{tabular}

$$
\begin{aligned}
C_{\bar{X}}^{ \pm}(t, \vec{p}=\overrightarrow{0})= & \frac{1}{2} \operatorname{Tr}\left(1 \pm \gamma_{0}\right) \\
& \times \sum_{\mathbf{x}_{\text {sink }}}\left\langle J_{X}\left(\mathbf{x}_{\text {sink }}, t_{\text {sink }}\right) \bar{J}_{X}\left(\mathbf{x}_{\text {source }}, t_{\text {source }}\right)\right\rangle, \\
t= & t_{\text {sink }}-t_{\text {source }} .
\end{aligned}
$$
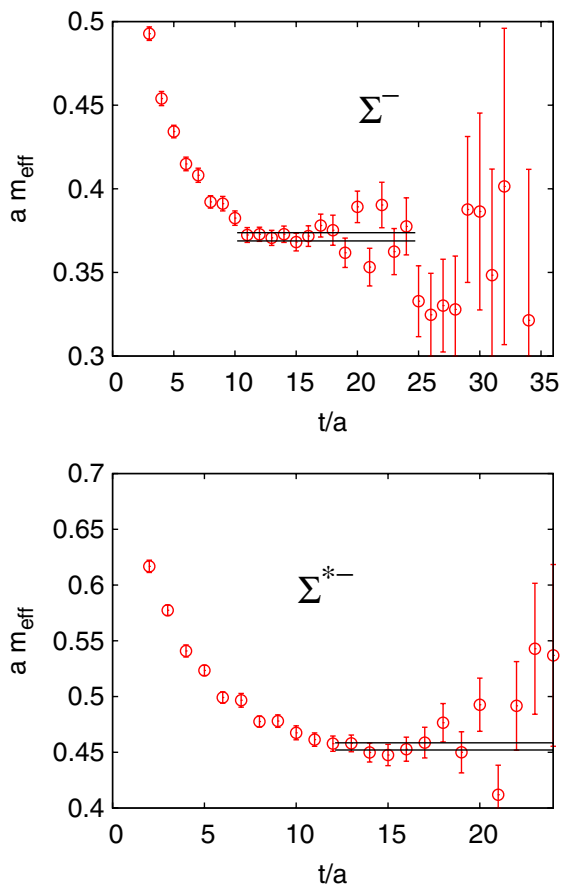

Space-time reflection symmetries of the action and the antiperiodic boundary conditions in the temporal direction for the quark fields imply, for zero three-momentum correlators, that $C_{X}^{+}(t)=-C_{X}^{-}(T-t)$. So, in order to decrease errors, we average correlators in the forward and backward directions and define
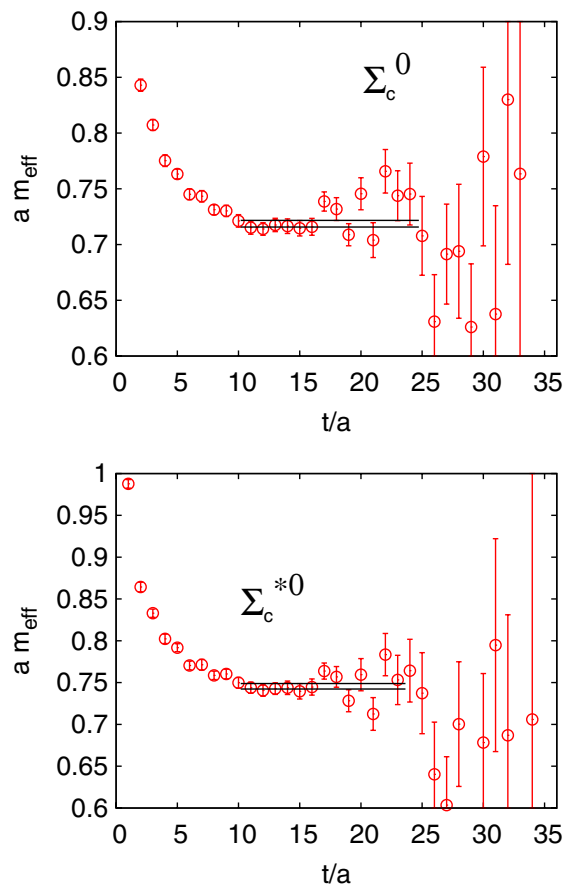

FIG. 5 (color online). Representative effective mass plots for $\beta=4.2$ and $a \mu_{l}=0.002$. For the strange baryons (left), we used $a \mu_{s}=0.015$, while for charm baryons (right), we used $a \mu_{c}=0.17$. 


$$
C_{X}(t)=C_{X}^{+}(t)-C_{X}^{-}(T-t) .
$$

In order to decrease correlation between measurements, we choose the source location randomly on the whole lattice for each configuration. Masses are then extracted from the so-called effective mass, which is defined by

$$
\begin{aligned}
m_{\mathrm{eff}}^{X}(t) & =-\log \left(C_{X}(t) / C_{X}(t-1)\right) \\
& =m_{X}+\log \left(\frac{1+\sum_{i=1}^{\infty} c_{i} e^{\Delta_{i} t}}{1+\sum_{i=1}^{\infty} c_{i} e^{\Delta_{i}(t-1)}}\right) \underset{t \rightarrow \infty}{\rightarrow} m_{X},
\end{aligned}
$$

where $\Delta_{i}=m_{i}-m_{X}$ is the mass difference of the excited state $i$ with respect to the ground mass $m_{X}$.

In Fig. 5, we show representative examples of the effective masses of strange and charm baryons. As can be seen, a plateau region can be clearly identified. What is shown in these figures are effective masses extracted from correlators where smearing is applied at both the sink and the source. Although local correlators are expected to have the same value in the large time limit, smearing suppresses excited state contributions, yielding a plateau at earlier time separations and to a better accuracy in the mass extraction. We therefore extract the masses using smeared sources and sinks. Our fitting procedure to extract $m_{X}$ is as follows: The sum over excited states in the effective mass given in Eq. (14) is truncated, keeping only the first excited state. Allowing a couple of time slice separations, the effective mass is fitted to the form given in Eq. (14). This yields an estimate for the parameters $c_{1}$ and $\Delta_{1}$. The lowerfit range is increased until the contribution due to the first excited state is less than $50 \%$ of the statistical error of $m_{X}$. This criterion is in most of the cases in agreement with a $\chi^{2} /$ d.o.f. $<1$. In the cases in which this criterion is not satisfied, a careful examination of the effective mass is made to ensure that the fit range is in the plateau region.

\section{LATTICE RESULTS}

Before we extrapolate our lattice results on the strange and charm baryon masses to the physical point, we need to examine their dependence on the heavy quark mass as well as cutoff effects. We collect lattice results for the masses of the strange and charm baryons in the Appendix. The errors
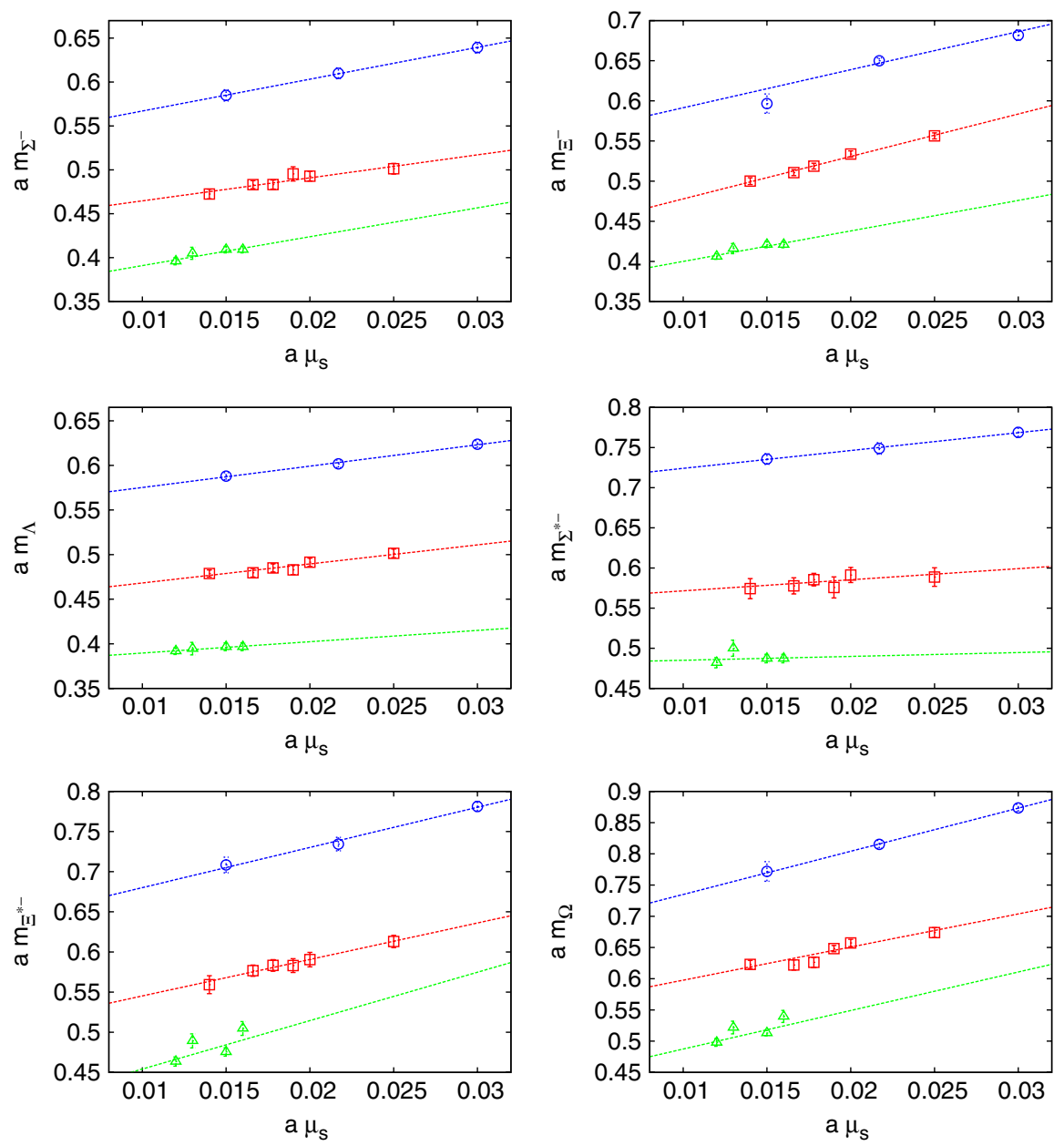

FIG. 6 (color online). Baryon masses versus the strange quark mass for $\beta=3.9$ and $a \mu_{l}=0.0064$ (circles), $\beta=4.05$ and $a \mu_{l}=$ 0.006 (squares), and $\beta=4.2$ and $a \mu_{l}=0.0065$ (triangles). 

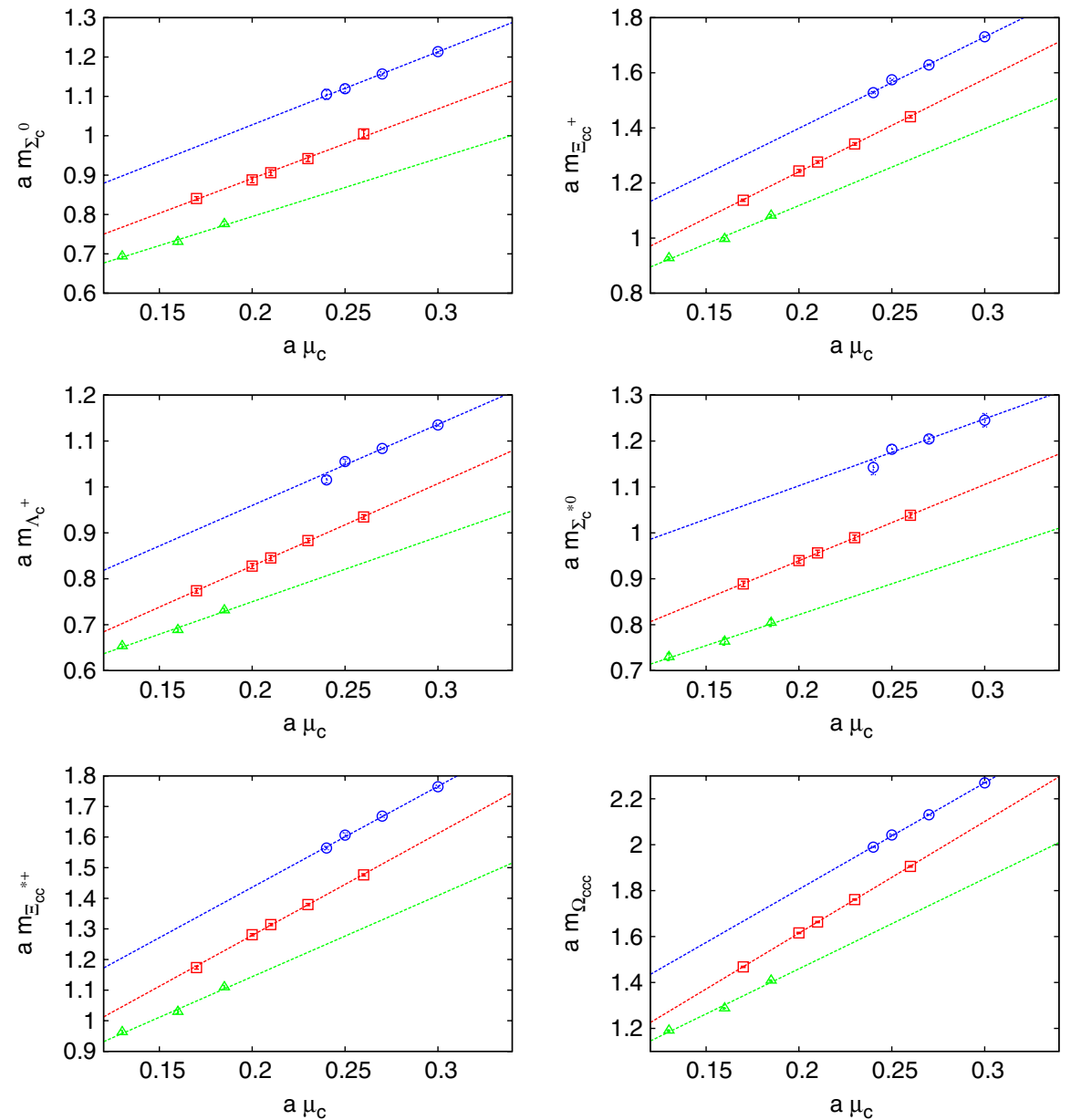

FIG. 7 (color online). Baryon masses versus the charm quark mass for $\beta=3.9$ and $a \mu_{l}=0.004$ (circles), $\beta=4.05$ and $a \mu_{l}=0.003$ (squares), and $\beta=4.2$ and $a \mu_{l}=0.0065$ (triangles).

are evaluated using jackknife and the $\Gamma$ method [34] to check consistency.

In Figs. 6 and 7, we show the dependence of the strange and charm baryon masses on the strange and charm quark masses, respectively. Overall, the data display a linear dependence on both the strange and charm quark mass. One can therefore interpolate between different values of quark masses, if needed.

\section{A. Strange baryon mass with strange quark mass tuned to its physical value}

In this subsection, we restrict our analysis only to the subset of data obtained at the tuned values of the strange quark mass. Namely, for $\beta=3.9$ and $\beta=4.05$, we use the tuned value given in Table III, whereas for $\beta=4.2$ we use $a \mu_{s}=0.015$, which agrees with the tuned strange quark mass within error bars.

It is interesting to examine the degree of isospin splitting as a function of the lattice spacing. The splitting is expected to be zero in the continuum limit. In Fig. 8, we show the masses of $\Sigma^{+}, \Sigma^{0}$, and $\Sigma^{-}$baryons at $\beta=3.9,4.05$, and 4.2. As expected, the mass splitting among the three charge states of the $\Sigma$ baryon decreases with the lattice spacing. The same behavior is observed for the other strange particles studied in this work. This is shown in Fig. 9, where we plot the mass difference as a function of $a^{2}$ at our smallest and heaviest pion masses. As can be seen, the mass difference is consistent with zero for all particles at the smallest lattice spacing. The small nonzero values seen for the $\Sigma$ and $\Xi$ particles are just outside one standard deviation. Therefore, the general conclusion is that isospin splitting is indeed small at these values of the lattice spacing, and it vanishes at the continuum limit. Since for finite $a$ there are small differences, for the chiral extrapolation where we use all lattice data, we do not average the masses for the different charge states of $\Sigma, \Xi$, and $\Xi^{*}$.

Volume effects can be studied at $\beta=3.9$, where we have simulations at two volumes for a pion mass of about $300 \mathrm{MeV}$. As can be seen in Figs. 8, 10, and 11, results at different volumes are consistent. Therefore, we conclude that any volume effects are smaller than our statistical accuracy.

In order to examine the continuum limit, we interpolate our lattice result at a given pion mass in units of $r_{0}$. 

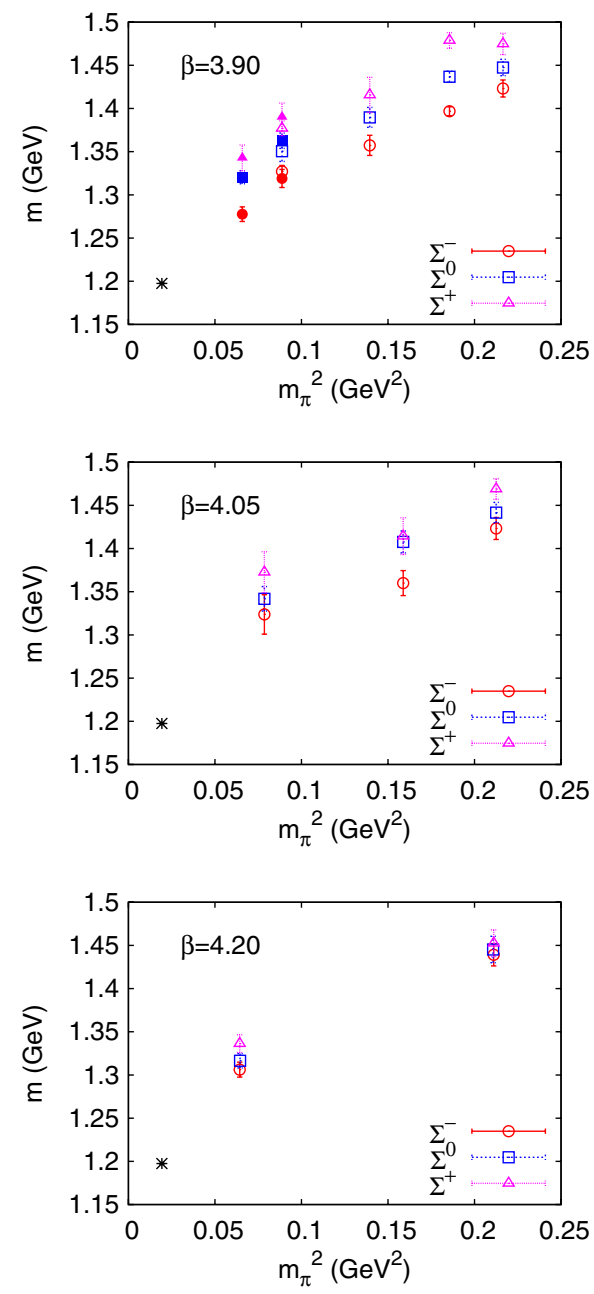

FIG. 8 (color online). The masses of $\Sigma^{+}, \Sigma^{0}$ and $\Sigma^{-}$baryons at $\beta=3.9,4.05$ and 4.2 . Filled symbols at $\beta=3.9$ refer to the larger volume.

For $\beta=4.2$ we have simulations at only two values of the pion mass, at the upper and lower range of pion masses considered in this work, namely for $m_{\pi}=0.254 \mathrm{GeV}$ and $m_{\pi}=0.459 \mathrm{GeV}$. Therefore, we interpolate the results for the other two values of $\beta$ to these two pion masses. In Figs. 10 and 11, we show results for the octet and decuplet strange baryon masses, respectively, at our three values of the lattice spacing. We perform a continuum extrapolation by performing a linear fit in $\left(a / r_{0}\right)^{2}$ as well as to a constant. As can be seen from Fig. 10, the values obtained in the continuum limit agree for all octet baryons. In the case of the decuplet, the statistical errors are larger and the value obtained at $a=0$ with the linear fit carries a large error. The value obtained using a constant fit has a smaller error and is compatible with the one obtained using a linear fit. Therefore, for a given charge state and within the current statistical accuracy, the $\left(a / r_{0}\right)^{2}$ term can be taken as negligible. Therefore, we can use results at all $\beta$ values to extrapolate to the physical point, since cutoffs are small for a given charge state. There are two exceptions in the case of the decuplet. At $\beta=4.05$, the masses of the $\Xi^{*}$ and $\Omega$ baryons at the lowest pion mass are systematically higher than at the other two $\beta$ values. Since the results at $\beta=3.9$ with larger lattice space are consistent with those at $\beta=4.2$, we conclude that this is not a cutoff effect.

\section{B. Charm baryon mass with charm quark mass tuned to its physical value}

As in the previous subsection, we consider results obtained at the tuned charm mass given in Table III. The only exception is at $\beta=4.2$ for the heavy pion mass, where we have results close to the tuned value, namely at $a \mu_{c}=0.16,0.185,0.21$. As we have seen, the dependence on the heavy quark is linear, and therefore the charm baryon masses at the tuned value can be easily determined by a linear interpolation.

We follow the same analysis as in the case of the strange baryon sector. In Fig. 12, we show the mass difference between different charged states as a function of the lattice spacing at the smallest and largest pion masses used in this work. As can be seen, the mass splittings are zero at the smallest value of the lattice spacing for all particles, confirming restoration of isospin symmetry in the continuum limit. Furthermore, except for the case of the $\Xi_{c c}$ mass, the mass splitting is also consistent with zero at the other two $\beta$ values. Therefore, for all particles except the $\Xi_{c c}$ baryon, one may average over the masses of different charge states.

In order to examine the continuum limit, we interpolate our results at the three $\beta$ values at a given pion mass in units of $r_{0}$. In Figs. 13 and 14, we show the masses in the octet and decuplet charm sector as a function of lattice spacing for a given charge state, at the smallest and largest values of the pion mass. A linear fit in $\left(a / r_{0}\right)^{2}$ and a constant fit yield consistent results at the continuum limit, albeit with large errors in the case of the linear fit. We also note that at the largest pion mass-although results at $\beta=3.9$ are in agreement with those at $\beta=4.2$, indicating negligible $\mathcal{O}\left(a^{2}\right)$ dependence-at $\beta=4.05$, the results are systematically lower. We note that we show only statistical errors. Systematic errors due, for example, to the matching are not shown. As discussed in the next section, these are 5-10\%. Therefore, a reasonable way to extrapolate our results in the charm sector is to compare the chiral extrapolation using all lattice data to those using results at $\beta=3.9$ and $\beta=4.2$. We will take the difference between the two values at the physical point as an estimate of a systematic error.

\section{CHIRAL EXTRAPOLATION}

Having determined that $\mathcal{O}\left(a^{2}\right)$ effects are small for the lattice spacings considered here, we can combine our lattice results at the various $\beta$ values to extrapolate to the physical pion mass (physical point). 

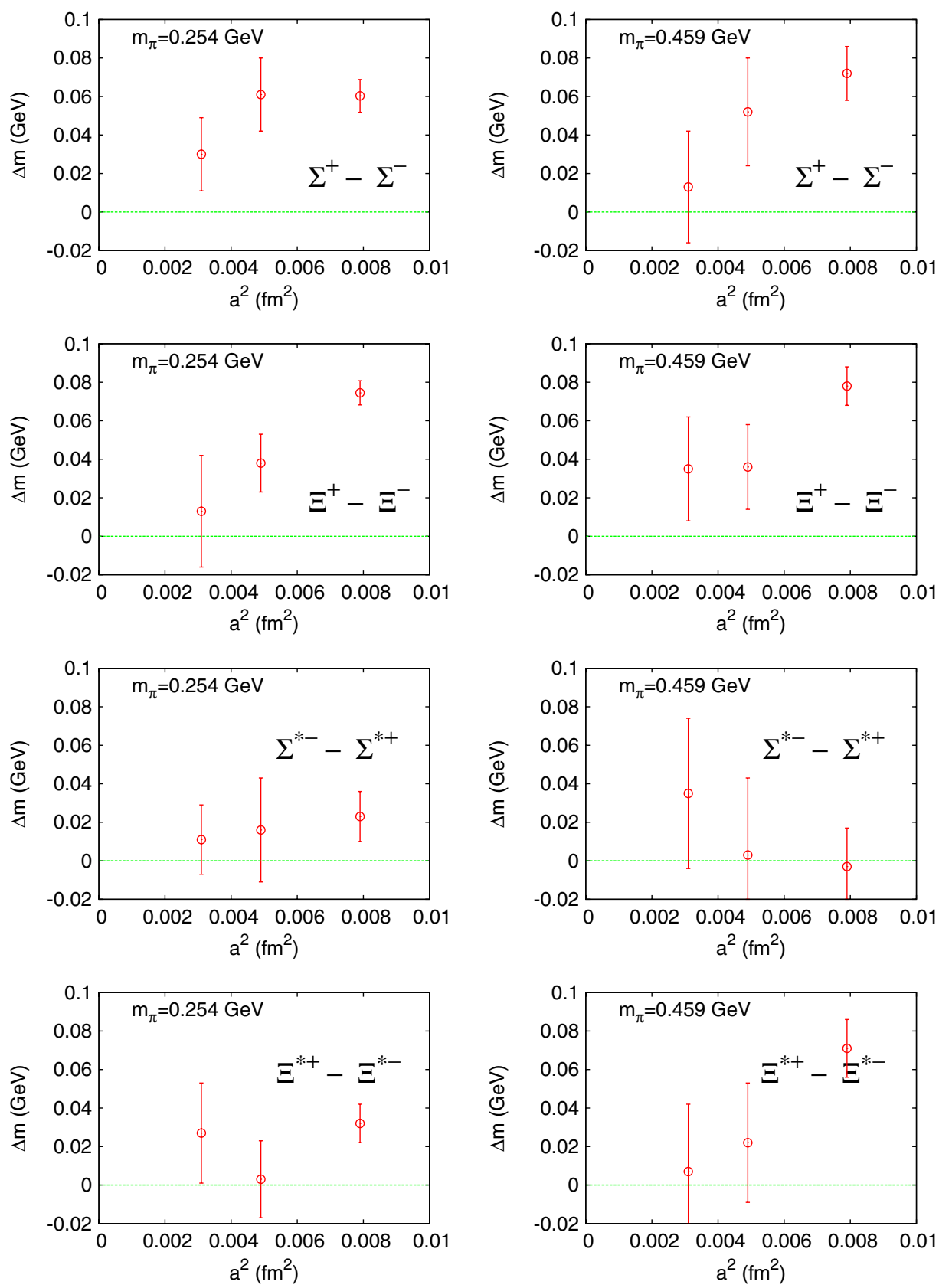

FIG. 9 (color online). Isospin mass splittings in the strange quark sector at the smallest (left) and largest (right) pion masses used in our computation.

For the strange baryon sector, we consider $S U(2)$ heavybaryon chiral perturbation, which has been found to describe lattice data satisfactorily [2]. To leading oneloop, one can describe the pion mass dependence using

$$
m_{B}=m_{B}^{(0)}-4 c_{B}^{(1)} m_{\pi}^{2}+c m_{\pi}^{3},
$$

where $c$ is a known coefficient given in Ref. [2]. For completeness, we give below the coefficients $c[35,36]$ for the octet baryons $\Lambda, \Sigma$, and $\Xi$ :

$$
c=-\frac{g_{\Lambda \Sigma}^{2}}{16 \pi f_{\pi}^{2}}, \quad-\frac{2 g_{\Sigma \Sigma}^{2}+g_{\Lambda \Sigma}^{2} / 3}{16 \pi f_{\pi}^{2}}, \quad-\frac{3 g_{\Xi \Xi}^{2}}{16 \pi f_{\pi}^{2}}
$$

respectively, and for the decuplet baryons $\Sigma^{*}, \Xi^{*}$, and $\Omega$ :

$$
c=-\frac{10}{9} \frac{g_{\Sigma^{*} \Sigma^{*}}^{2}}{16 \pi f_{\pi}^{2}}, \quad-\frac{5}{3} \frac{g_{\Xi^{*} \Xi^{*}}^{2}}{16 \pi f_{\pi}^{2}} m_{\pi}^{3}, \quad 0 .
$$

In addition, we consider next-to-leading-order (NLO) $S U(2) \chi \mathrm{PT}$ results [37]. The expressions are included here for completeness: 

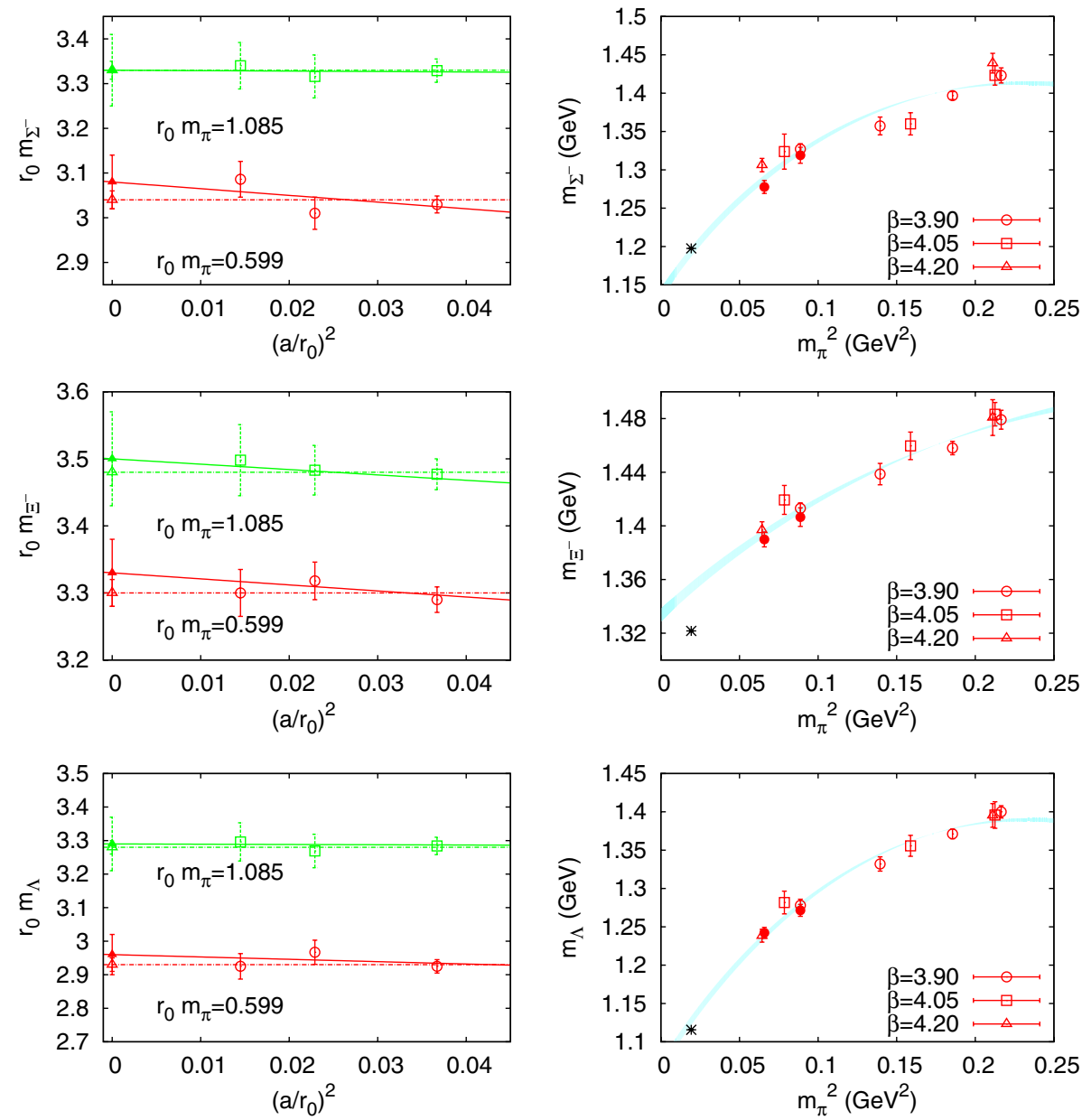

FIG. 10 (color online). Left: The strange octet baryon mass in units of $r_{0}$ versus $\left(a / r_{0}\right)^{2}$ for several particles in the octet and decuplet at two fixed values of $m_{\pi} r_{0}$. The solid line is a linear fit in $\left(a / r_{0}\right)^{2}$, and the dashed line is a fit to a constant. Right: Chiral extrapolation at fixed strange quark mass. Results at $\beta=3.9$ are shown with open circles for $L=2.1 \mathrm{fm}$ and with filled circles for $L=2.7$ fm; results at $\beta=4.05$ are shown with open squares, and results at $\beta=4.2$ with open triangles.

$$
\begin{aligned}
& m_{\Lambda}^{\mathrm{NLO}}\left(m_{\pi}\right)=m_{\Lambda}^{(0)}-4 c_{\Lambda}^{(1)} m_{\pi}^{2}-\frac{g_{\Lambda \Sigma}^{2}}{\left(4 \pi f_{\pi}\right)^{2}} \mathcal{F}\left(m_{\pi}, \Delta_{\Lambda \Sigma}, \lambda\right)-\frac{4 g_{\Lambda \Sigma^{*}}^{2}}{\left(4 \pi f_{\pi}\right)^{2}} \mathcal{F}\left(m_{\pi}, \Delta_{\Lambda \Sigma^{*}}, \lambda\right), \\
& m_{\Sigma}^{\mathrm{NLO}}\left(m_{\pi}\right)=m_{\Sigma}^{(0)}-4 c_{\Sigma}^{(1)} m_{\pi}^{2}-\frac{2 g_{\Sigma \Sigma}^{2}}{16 \pi f_{\pi}^{2}} m_{\pi}^{3}-\frac{g_{\Lambda \Sigma}^{2}}{3\left(4 \pi f_{\pi}\right)^{2}} \mathcal{F}\left(m_{\pi},-\Delta_{\Lambda \Sigma}, \lambda\right)-\frac{4 g_{\Lambda \Sigma^{*}}^{2}}{3\left(4 \pi f_{\pi}\right)^{2}} \mathcal{F}\left(m_{\pi}, \Delta_{\Sigma \Sigma^{*}}, \lambda\right), \\
& m_{\Xi}^{\mathrm{NLO}}\left(m_{\pi}\right)=m_{\Xi}^{(0)}-4 c_{\Xi}^{(1)} m_{\pi}^{2}-\frac{3 g_{\Xi \Xi}^{2}}{16 \pi f_{\pi}^{2}} m_{\pi}^{3}-\frac{2 g_{\Xi^{*} \Xi}^{2}}{\left(4 \pi f_{\pi}\right)^{2}} \mathcal{F}\left(m_{\pi}, \Delta_{\Xi \Xi^{*}}, \lambda\right),
\end{aligned}
$$

and for the decuplet baryons:

$$
\begin{aligned}
& m_{\Sigma^{*}}^{\mathrm{NLO}}\left(m_{\pi}\right)=m_{\Sigma^{*}}^{(0)}-4 c_{\Sigma^{*}}^{(1)} m_{\pi}^{2}-\frac{10}{9} \frac{g_{\Sigma^{*} \Sigma^{*}}^{2}}{16 \pi f_{\pi}^{2}} m_{\pi}^{3}-\frac{2}{3\left(4 \pi f_{\pi}\right)^{2}}\left[g_{\Sigma^{*} \Sigma}^{2} \mathcal{F}\left(m_{\pi},-\Delta_{\Sigma \Sigma^{*}, \lambda}\right)+g_{\Lambda \Sigma^{*}}^{2} \mathcal{F}\left(m_{\pi},-\Delta_{\Lambda \Sigma^{*}, \lambda}\right)\right], \\
& m_{\Xi^{*}}^{\mathrm{NLO}}\left(m_{\pi}\right)=m_{\Xi^{*}}^{(0)}-4 c_{\Xi^{*}}^{(1)} m_{\pi}^{2}-\frac{5}{3} \frac{g_{\Xi^{*} \Xi^{*}}^{2}}{16 \pi f_{\pi}^{2}} m_{\pi}^{3}-\frac{g_{\Xi^{*}}^{2} \Xi}{\left(4 \pi f_{\pi}\right)^{2}} \mathcal{F}\left(m_{\pi},-\Delta_{\Xi \Xi^{*}, \lambda}\right), \\
& m_{\Omega}^{\mathrm{NLO}}\left(m_{\pi}\right)=m_{\Omega}^{(0)}-4 c_{\Omega}^{(1)} m_{\pi}^{2},
\end{aligned}
$$

with the nonanalytic function [38] 

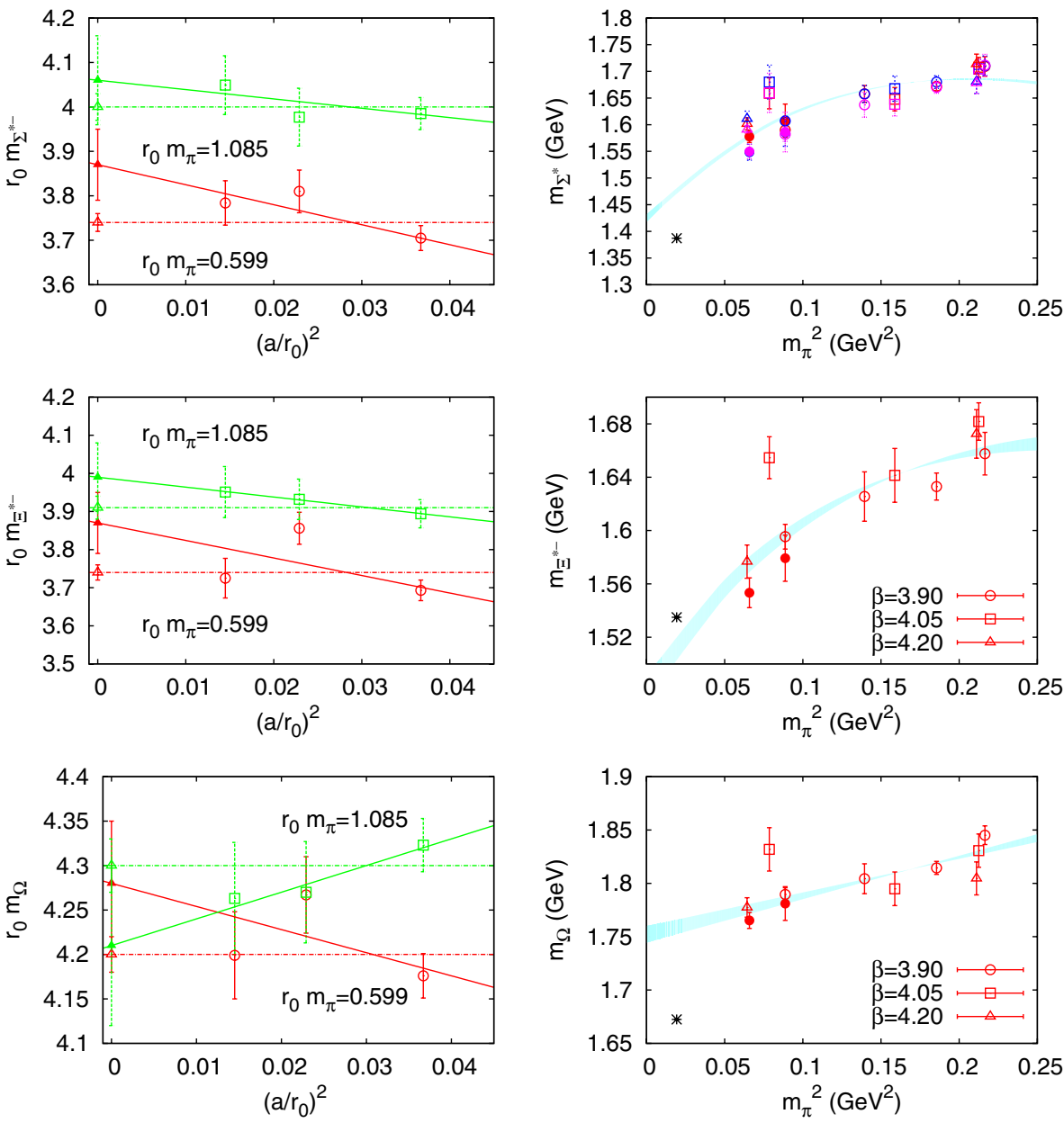

FIG. 11 (color online). Left: The strange decuplet baryon mass in units of $r_{0}$ versus $\left(a / r_{0}\right)^{2}$ at two fixed values of $m_{\pi} r_{0}$. The solid line is a linear fit in $\left(a / r_{0}\right)^{2}$, and the dashed line is a fit to a constant. Right: Chiral extrapolation at fixed strange quark mass. In the case of the $\Sigma^{*}$ baryon, we show the three charge states $\Sigma^{*+}$ (purple), $\Sigma^{* 0}$ (blue), and $\Sigma^{*-}$ (red). The spread seen in the data with the same symbol at a given value of the pion mass indicates the level of isospin breaking for the $\Sigma^{*}$ particle. Results at $\beta=3.9$ are shown with open circles for $L=2.1 \mathrm{fm}$ and with filled circles for $L=2.7 \mathrm{fm}$; results at $\beta=4.05$ are shown with open squares, and results at $\beta=4.2$ with open triangles.

$\mathcal{F}(m, \Delta, \lambda)$

$$
\begin{aligned}
= & \left(m^{2}-\Delta^{2}\right) \sqrt{\Delta^{2}-m^{2}+i \epsilon} \log \left(\frac{\Delta-\sqrt{\Delta^{2}-m^{2}+i \epsilon}}{\Delta+\sqrt{\Delta^{2}-m^{2}+i \epsilon}}\right) \\
& -\frac{3}{2} \Delta m^{2} \log \left(\frac{m^{2}}{\lambda^{2}}\right)-\Delta^{3} \log \left(\frac{4 \Delta^{2}}{m^{2}}\right),
\end{aligned}
$$

depending on the threshold parameter $\Delta_{X Y}=m_{Y}^{(0)}-m_{X}^{(0)}$ and on the scale $\lambda$ of chiral perturbation theory, fixed to $\lambda=1 \mathrm{GeV}$. For $\Delta>0$, the real part of the function $\mathcal{F}(m, \Delta, \lambda)$ has the property

$\mathcal{F}(m,-\Delta, \lambda)=\left\{\begin{array}{ll}-\mathcal{F}(m, \Delta, \lambda) & m<\Delta \\ -\mathcal{F}(m, \Delta, \lambda)+2 \pi\left(m^{2}-\Delta^{2}\right)^{3 / 2} & m>\Delta\end{array}\right.$, which corrects a typo in the sign of the second term in Ref. [5]. We follow the procedure of Ref. [2] and fix the nucleon axial charge $g_{A}$ and pion decay constant $f_{\pi}$ to their experimental values (we use the convention such that $f_{\pi}=130.70 \mathrm{MeV}$ ). The remaining pion-baryon axial coupling constants are taken from $S U(3)$ relations [37]. The fit parameters extracted for fitting to the NLO are given in Table VII. The deviation of the mean values obtained at the physical point when the results are fitted to leading order-i.e., to Eq. (15) with $c=0$-and when they are fitted to the NLO expressions provide an estimate of the systematic error due to the chiral extrapolation. We give this error in Table VII. In the case of the $\Omega$ baryon, there is no difference between leading order (LO) and nextto-leading order. Since the $\Omega$ baryon contains three strange quarks, any systematic error in the tuning of the strange quark mass will be the largest in this case. Having results at 

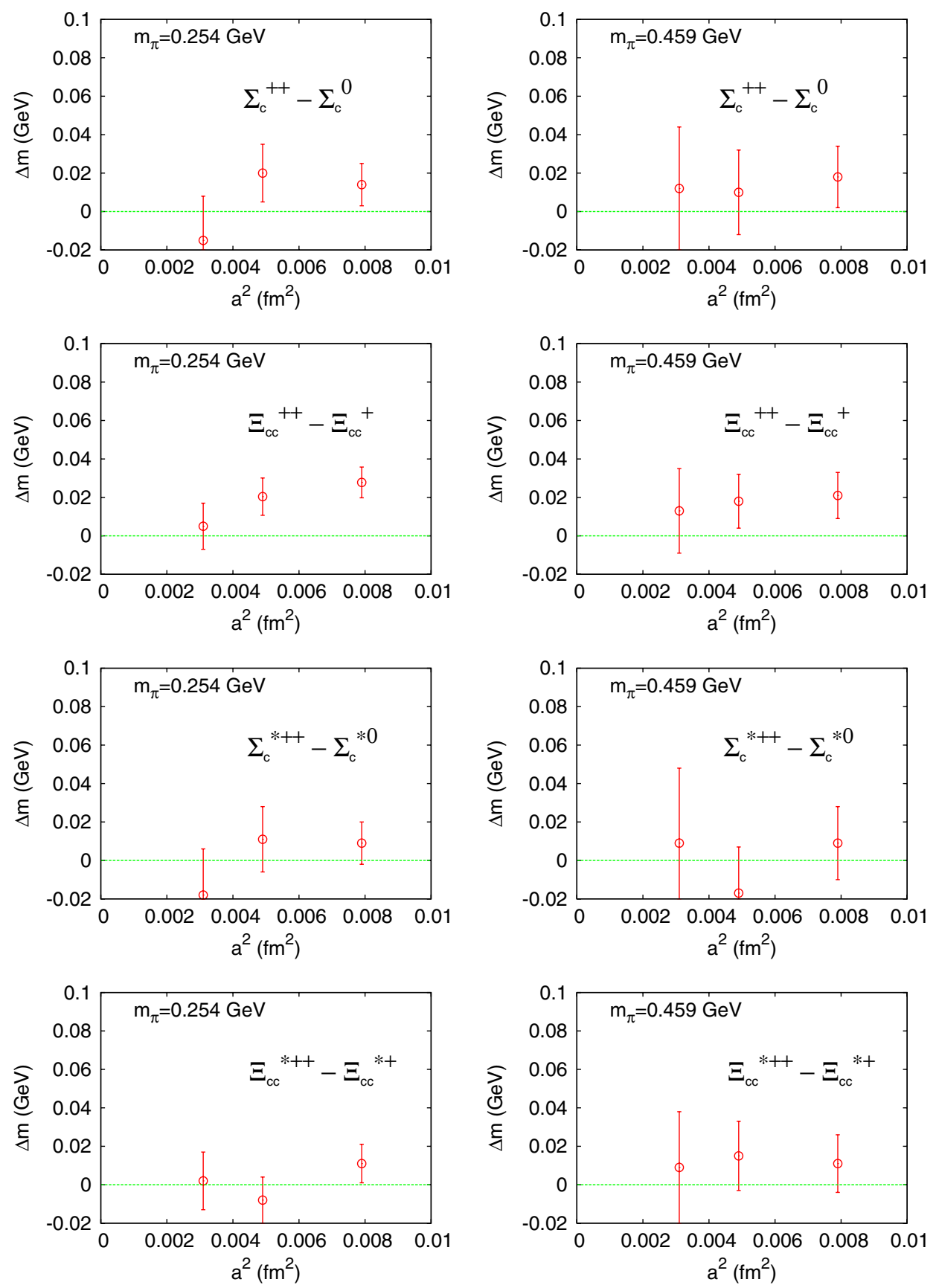

FIG. 12 (color online). Isospin mass splitting in the charm sector at the smallest and largest values of the pion mass used in this work.

several values of the strange quark mass, we can estimate the change in the $\Omega$ mass if the strange quark mass takes the maximum and minimum values allowed by the statistical error in the tuned strange quark mass. We take the difference in the mean values at the physical point obtained by varying the strange quark mass to be the systematic error due to the tuning. In Table VII, we give the systematic error on the mass of $\Omega$ that we find following this procedure. This gives an upper bound of the error expected from the uncertainty in the tuning. As can be seen, this is smaller as compared to the systematic error due to the chiral extrapolation, and therefore it is only taken into account for the case of the $\Omega$ baryon.

In Figs. 10 and 11, we show the chiral extrapolation for the octet and the decuplet. In the cases of the $\Sigma$ and $\Xi^{*}$ baryons, the physical point is reproduced. However, for most other particles, the lattice results extrapolate to a higher value. The worse deviation is seen for the $\Omega$ baryon. Since this has three strange quarks, it may indicate that the tuning of the strange quark mass performed using the kaon mass introduces a systematic error. One can study partial quenching effects using twisted mass fermion simulations 

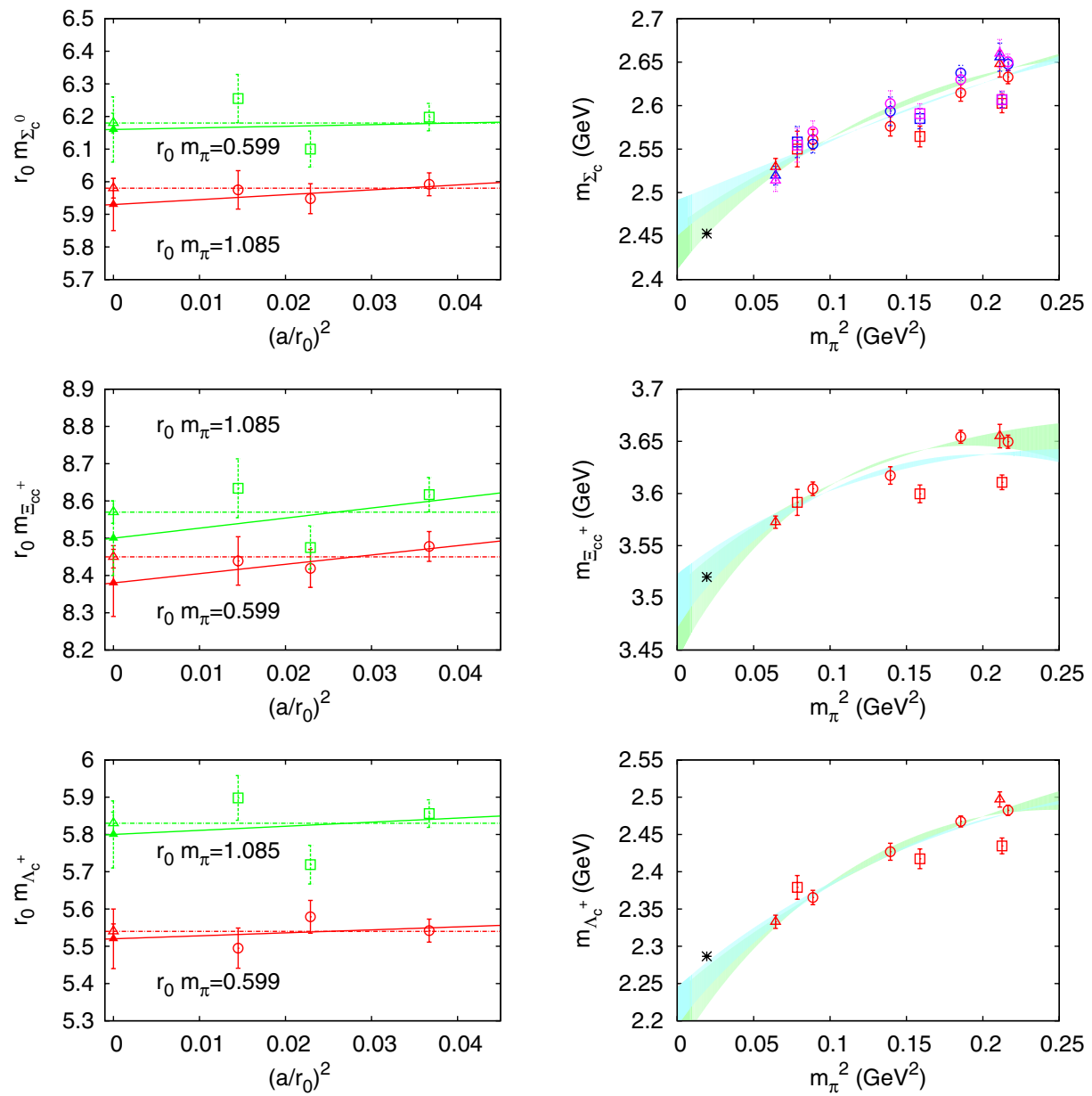

FIG. 13 (color online). Left: The masses of octet charm baryons in units of $r_{0}$ versus $\left(a / r_{0}\right)^{2}$ for several particles in the octet and decuplet at two fixed values of the pion mass $m_{\pi} r_{0}$. Right: Chiral fits to the data at $\beta=3.9$ (circles) and at $\beta=4.2$ (triangle) are shown with the green band; fits to all data are shown with the blue band. For the $\Xi_{c c}$ baryon we have extrapolated $\Xi_{c c}^{+}$, whereas for the $\Sigma_{c}$ baryon we averaged over $\Sigma_{c}^{++}$(purple), $\Sigma_{c}^{+}$(blue) and $\Sigma_{c}^{0}$ (red). The spread seen in the data with the same symbol at a given value of the pion mass indicates the level of isospin breaking for the $\Sigma_{c}$ particle.

with a dynamical strange quark. This will be considered in a future study.

In the charm baryon sector, we use the ansatz

$$
m_{B}=a+b m_{\pi}^{2}+c m_{\pi}^{3},
$$

motivated by $S U(2) \mathrm{HB} \chi \mathrm{PT}$ to leading one-loop order, with $c$ taken as a fit parameter. For the $\Omega_{c c c}$ baryon, we set $c=0$, since one does not expect a cubic term.

In order to assess the systematic error associated with the tuning of the charm quark mass, we consider our results at $\beta=3$.9. At this value of $\beta$, we have computed the charm baryon masses at the tuned value of the charm quark and at values of the charm quark shifted by the error on the tuned value. Since these computations were performed at four different light quark masses, we can perform a chiral extrapolation using the ansatz of Eq. (22) for the set of masses obtained at the tuned value and at the value shifted by the error. The difference in the masses obtained at the physical pion mass is given in Table VIII. As can be seen, this difference introduces an error that varies between about $5 \%$ and $10 \%$. This gives us an estimate of the systematic error due to the tuning of the charm quark mass. Since this analysis can only be done at $\beta=3.9$, we can only make a qualitative estimate of this error. Therefore, in what follows, we will not quote this error on our values. However, one has to bear in mind that our final values can have a systematic error of about $10 \%$ due to the tuning.

In Figs. 13 and 14, we show fits for our three $\beta$ values. We show fits using all data and fits using only data at $\beta=3.9$ and $\beta=4.2$. The latter case yields a better fit with a smaller value of $\chi /$ d.o.f., and this is the value quoted in Table IX. This is particularly noticeable for the case of $\Omega_{c c c}$ baryons, where the results at $\beta=4.05$ are systematically lower. This may be due to a small mismatch in the tuned value of the charm quark mass, which for the $\Omega_{c c c}$ baryon, that contains three charm quarks, would lead to the largest deviation. We take the difference in the extrapolated values at the physical points when we exclude the $\beta=4.05$ data from the fit as a systematic error. 

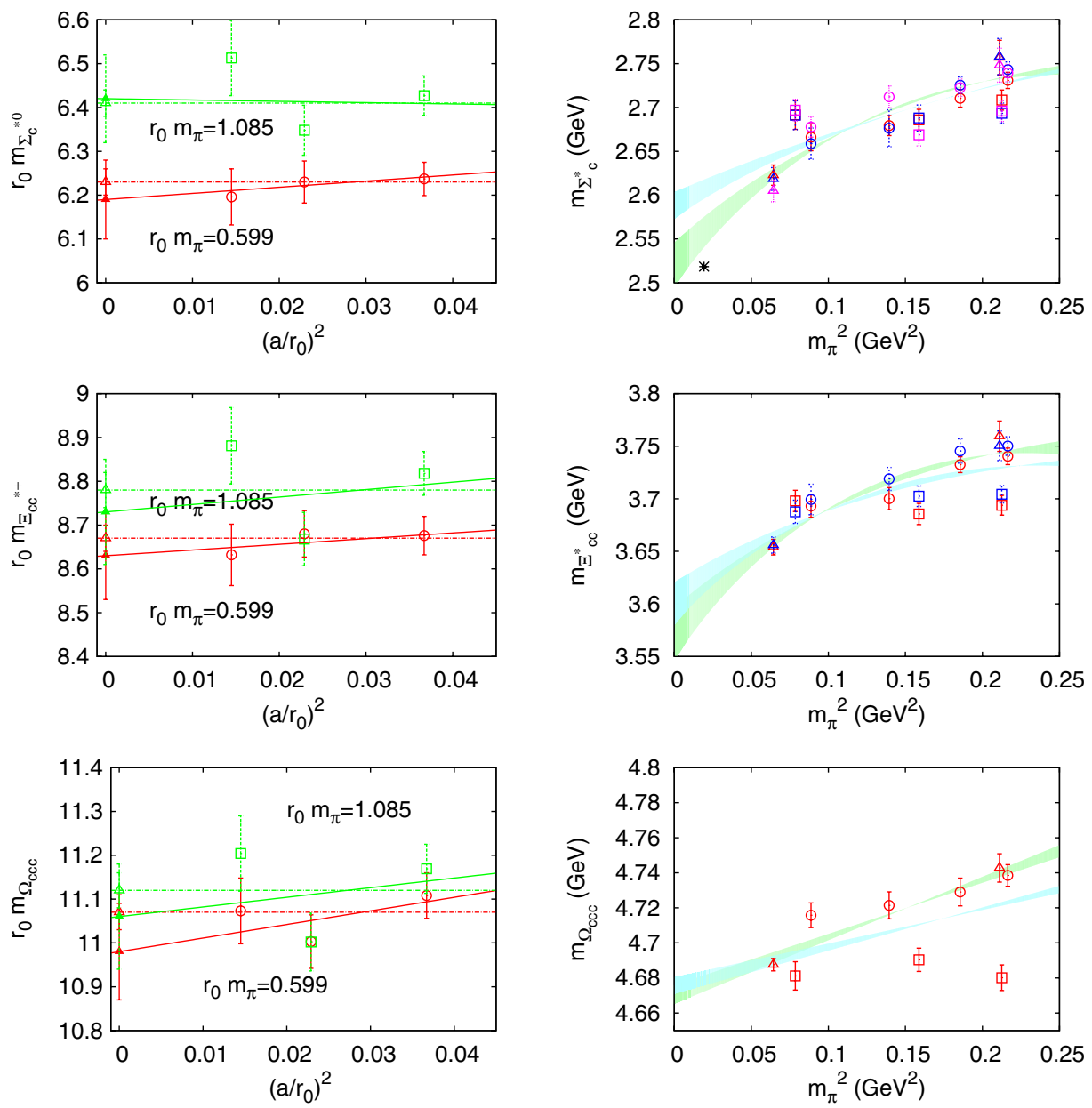

FIG. 14 (color online). Left: The masses of decuplet charm baryons in units of $r_{0}$ versus $\left(a / r_{0}\right)^{2}$ at two fixed values of $m_{\pi} r_{0}$. Right: Chiral fits to the data at $\beta=3.9$ (circles) and at $\beta=4.2$ (triangle) are shown with the green band; fits to all data are shown with the blue band. For the $\Xi_{c c}^{*}$ baryon we averaged over $\Xi_{c c}^{*++}$ (blue) and $\Xi_{c c}^{*+}$ (red), and for the $\Sigma_{c}^{*}$ baryon we averaged over $\Sigma_{c}^{*++}$ (purple), $\Sigma_{c}^{*+}$ (blue) and $\Sigma_{c}^{* 0}$ (red). The spread seen in the data with the same symbol at a given value of the pion mass indicates the level of isospin breaking for the or the $\Sigma_{c}^{*}$ and $\Xi_{c}^{*}$ particles.

TABLE VII. The bare mass and $c_{B}$ (related to the $\sigma$ term by $\sigma_{B}=-4 c_{B} m_{\pi}^{2}$ ) determined from fitting to the NLO expressions for strange baryons at the tuned strange quark mass. In the last column, we give the mass in $\mathrm{GeV}$ that we obtain at the physical point using the NLO expressions. The error given in the second parenthesis is an estimate of the systematic error coming from a comparison between the values obtained at the physical point using the LO expressions given in Eqs. (16) and (17) and the NLO expressions given by Eqs. (18) and (19). In the case of the $\Omega$ baryon, the systematic error is estimated by evaluating the impact of the error of the tuned strange quark mass on the extrapolated $\Omega$ mass.

\begin{tabular}{lcccc}
\hline \hline Particle(PDG) & $m_{B}^{(0)}(\mathrm{GeV})$ & $-4 c_{B}^{(1)}\left(\mathrm{GeV}^{-1}\right)$ & $\chi^{2} /$ d.o.f. & $m(\mathrm{GeV})$ \\
\hline$\Sigma^{-}(1193)$ & $1.1368(70)$ & $3.560(40)$ & 2.7 & $1.1930(62)(660)$ \\
$\Xi^{-}(1315)$ & $1.3334(46)$ & $1.386(26)$ & 0.82 & $1.3538(41)(179)$ \\
$\Lambda(1116)$ & $1.0678(64)$ & $4.362(37)$ & 1.04 & $1.1276(57)(721)$ \\
$\Sigma_{a v}^{*}(1384)$ & $1.4244(58)$ & $2.807(34)$ & 2.4 & $1.4757(51)(740)$ \\
$\Xi^{*-}(1531)$ & $1.4808(96)$ & $1.582(58)$ & 3.3 & $1.5113(89)(400)$ \\
$\Omega(1673)$ & $1.7522(76)$ & $0.361(45)$ & 2.0 & $1.7591(67)(200)$ \\
\hline \hline
\end{tabular}

The extrapolation of the lattice data reproduces experimentally measured charm baryon masses within a standard deviation, namely the masses of the $\Sigma_{c}, \Lambda_{c}$, and $\Sigma_{c}^{*}$ baryons. Therefore, the extrapolated lattice value can be taken as a

TABLE VIII. For each particle listed in the first column, we give in the second column its mass at the physical pion mass, using for the chiral extrapolation the masses computed at the tuned value of the charm quark mass $m_{c}$. In the third column, we give the mass difference between the baryon masses obtained at the tuned value of $m_{c}$ and at the tuned value plus the error, after extrapolation to the physical point. This is done at $\beta=3.9$ where we have computed the masses at $m_{c} \pm$ error.

\begin{tabular}{lcc}
\hline \hline Particle(PDG) & $m(\mathrm{GeV})$ & $\Delta m(\mathrm{GeV})$ \\
\hline$\Sigma_{c, a v}(2.454)$ & $2.494(47)$ & 0.143 \\
$\Xi_{c c}^{+}$ & $3.563(25)$ & 0.397 \\
$\Lambda_{c}^{+}(2286)$ & $2.229(43)$ & 0.223 \\
$\Sigma_{c, a v}^{*}(2.520)$ & $2.650(39)$ & 0.147 \\
$\Xi_{c c, a v}^{*}$ & $3.672(42)$ & 0.274 \\
$\Omega_{c c c}$ & $4.702(11)$ & 0.308 \\
\hline \hline
\end{tabular}


TABLE IX. Parameters of the chiral fit for charm baryons at the tuned charm quark mass, fitting results at $\beta=3.9$ and $\beta=4.2$. The last column is our prediction (in $\mathrm{GeV}$ ) of the mass at the physical point. The statistical error is given in the first parenthesis, and the systematic error, computed by comparing the fit with all lattice data, is given in the second parenthesis.

\begin{tabular}{llcccc}
\hline \hline Particle (PDG) & $m_{B}^{0}(\mathrm{GeV})$ & $-4 c_{B}\left(\mathrm{GeV}^{-1}\right)$ & $c\left(\mathrm{GeV}^{-2}\right)$ & $\chi^{2} /$ d.o.f. & $m(\mathrm{GeV})$ \\
\hline$\Sigma_{c, a v}(2.454)$ & $2.437(25)$ & $1.92(54)$ & $-2.09(91)$ & 1.1 & $2.468(17)(23)$ \\
$\Xi_{c c}^{+}$ & $3.476(35)$ & $2.39(83)$ & $-3.39(1.5)$ & 2.7 & $3.513(23)(14)$ \\
$\Lambda_{c}^{+}(2286)$ & $2.198(40)$ & $2.99(96)$ & $-3.6(1.7)$ & 0.10 & $2.246(27)(15)$ \\
$\Sigma_{c, a v}^{*}(2.520)$ & $2.520(25)$ & $2.37(51)$ & $-2.96(86)$ & 1.3 & $2.556(18)(51)$ \\
$\Xi_{c c, a v}^{*}$ & $3.571(25)$ & $2.02(57)$ & $-2.62(99)$ & 1.0 & $3.603(17)(21)$ \\
$\Omega_{c c c}^{*}$ & $4.6706(53)$ & $0.327(35)$ & 0 & 2.5 & $4.6769(46)(30)$ \\
\hline \hline
\end{tabular}

prediction for the masses of the $\Xi_{c c}^{*}$ and $\Omega_{c c c}$ baryons within one standard deviation.

\section{COMPARISON WITH THE RESULTS OF OTHER LATTICE FORMULATIONS}

In this section, we compare our results with those using different discretization schemes by other collaborations. We also include a comparison for the nucleon and $\Delta$ masses, although they were not discussed in detail until now.

Several collaborations have calculated the strange baryon spectrum. The Budapest-Marseille-Wuppertal (BMW) Collaboration carried out simulations using tree-levelimproved six-step stout smeared $N_{f}=2+1$ clover fermions and a tree-level Symanzik improved gauge action. Volume effects were studied using lattices of spatial extent from $2 \mathrm{fm}$ to $4.1 \mathrm{fm}$. The continuum limit was taken using results produced on three lattice spacings of $a=0.065 \mathrm{fm}$, $a=0.085 \mathrm{fm}$, and $a=0.125 \mathrm{fm}$. Using pion masses down to $190 \mathrm{MeV}$, a polynomial was performed to extrapolate to the physical value of the pion mass [4]. The PACS-CS Collaboration obtained results using $N_{f}=2+1$ nonperturbatively $\mathcal{O}(a)$-improved clover fermions on an Iwasaki gauge action on a lattice with a spatial length of $2.9 \mathrm{fm}$ and lattice spacing $a=0.09 \mathrm{fm}$ [3]. The QCDSF-UKQCD Collaboration [39] used $N_{f}=2+1$ Clover fermions with a single mild stout smearing and a lattice spacing of $a=$ 0.076(2) fm. Finally, the LPHC Collaboration [5] obtained results using a hybrid action of domain-wall valence quarks on a staggered sea on lattices of spatial length $2.5 \mathrm{fm}$ and $3.5 \mathrm{fm}$ at a lattice spacing of $a=0.124 \mathrm{fm}$.

In Fig. 15, we compare our results on the strange octet baryons with those from the BMW, the PACS-CS, the QCDSF-UKQCD and the LHPC collaborations. Our results and the results by the PACS-CS and LHPC are not
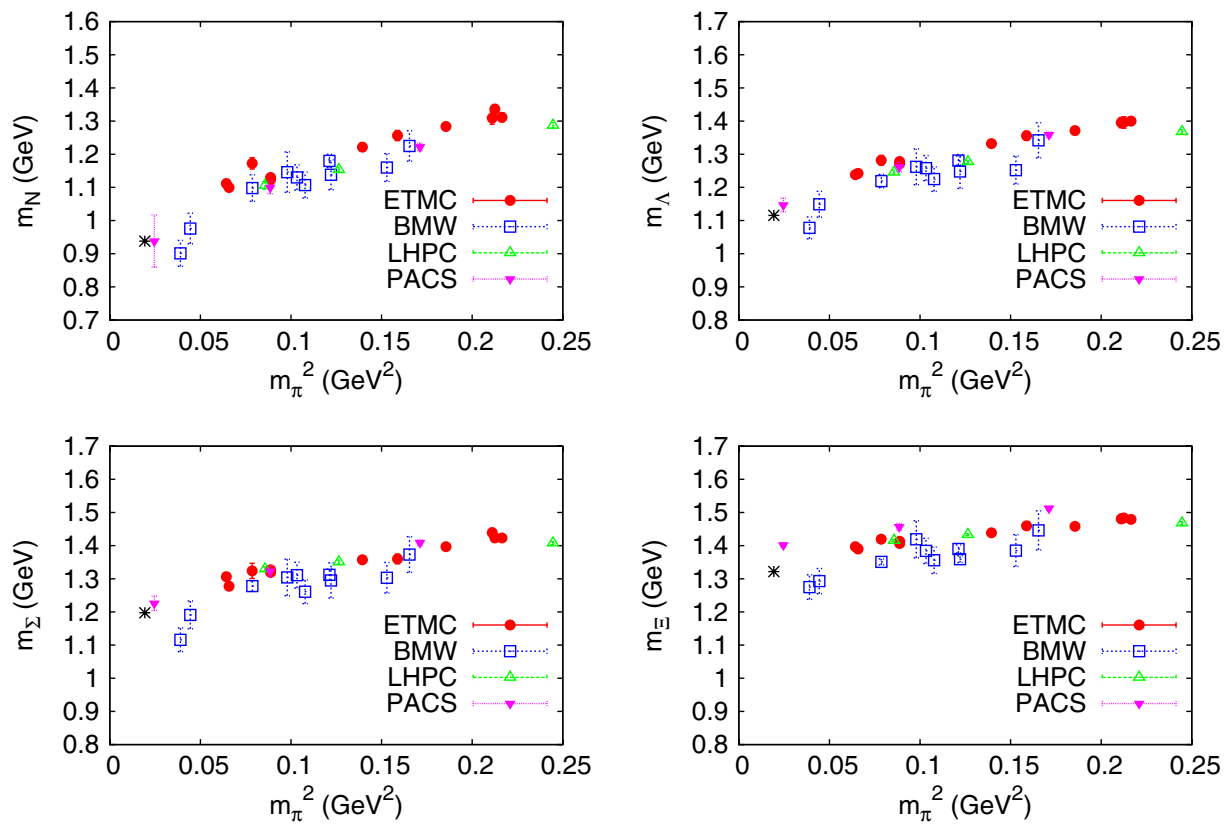

FIG. 15 (color online). The results of this work for the octet strange baryons are shown with filled (red) circles, results by the BMW Collaboration using clover fermions are shown with the open (blue) square [4], results by the PACS-CS Collaboration are shown with filled (magenta) triangles [3], and results by the LHPC using domain-wall valence quarks on a staggered sea are shown with open (green) triangles [5]. The experimental value is shown with an asterisk. 

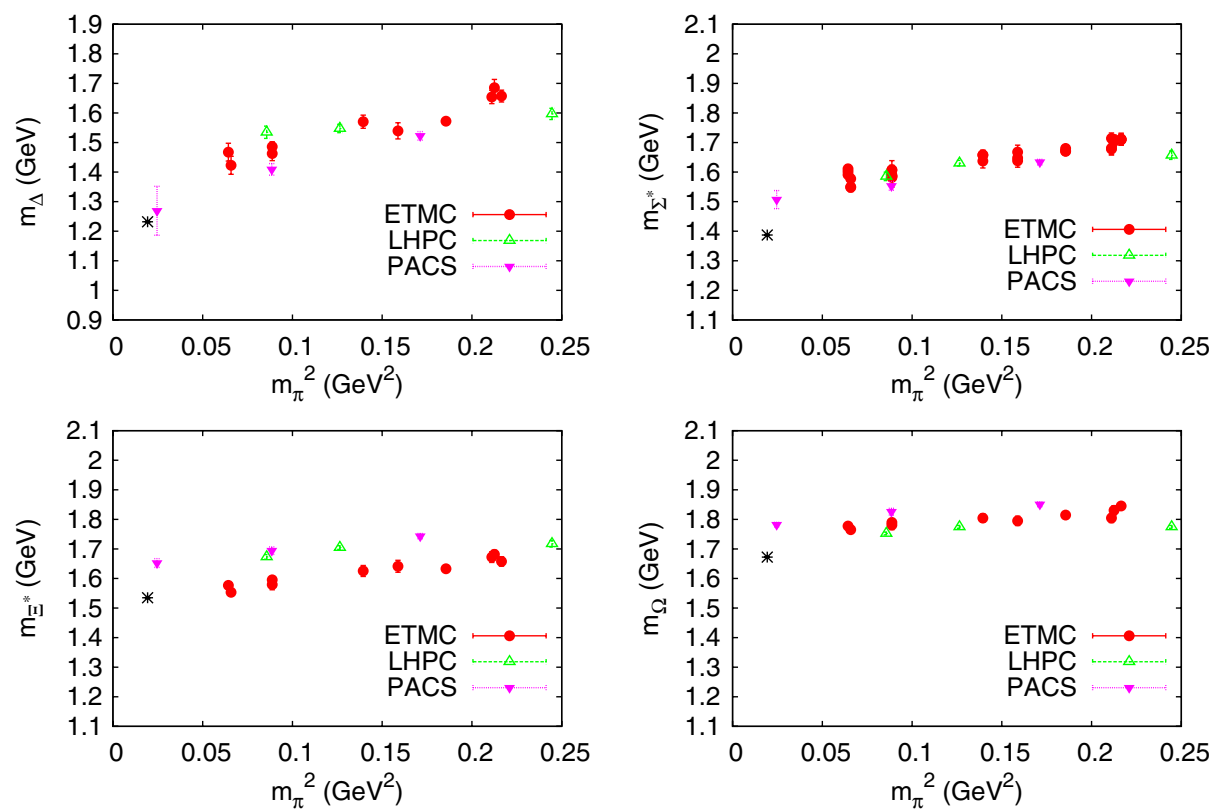

FIG. 16 (color online). Comparison of the results of this work for the strange decuplet baryons to those obtained using domain wall valence quarks on a staggered sea [5]. The notation is the same as that in Fig. 15.

continuum extrapolated. The BMW results are extrapolated to the continuum limit and have larger errors than the rest. Nevertheless, there is an overall agreement, indicating that cutoff effects are small. In Fig. 16, we compare our results on the strange decuplet baryons with the ones by PACS-CS and LHPC. Lattice results are in agreement except for the case of the $\Xi^{*}$ baryon, where our results are consistently lower. Given the agreement of our results in the case of the $\Omega$ baryon, this deviation cannot come from the mismatch in the strange quark mass. It is not clear what is the origin of this deviation for the $\Xi^{*}$ baryon, but the fact that the value obtained by PACS-CS at almost physical pion mass is higher than the experimental value may indicate that the strange quark mass is larger than physical. In Fig. 17, we show the

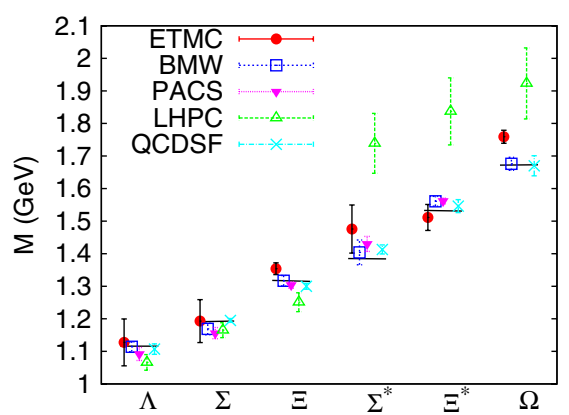

FIG. 17 (color online). Lattice data in the strange quark sector extrapolated to the physical pion mass, with the experimental values shown by the horizontal lines. For the twisted mass results of this work, the chiral extrapolation was carried out using NLO $\mathrm{HB} \chi \mathrm{PT}$. We include in addition the results by the QCDSFUKQCD Collaboration [39] (light blue crosses). The rest of the notation is the same as in Fig. 15. masses for the strange baryons after extrapolating to the physical pion mass. For the results of this work, we plot the values extracted using NLO HB $\chi \mathrm{PT}$. Error shown on the twisted mass results is the estimate of the systematic error due to the chiral extrapolation, whereas the statistical errors are equal to the sizes of the symbols and are not shown. As can be seen, our results are in agreement with experiment except for the $\Omega$ baryon, which is higher by $2 \%$, just like the value found by PACS-CS.

We also compare in Fig. 18 our results for the charm baryons to those obtained using dynamical gauge configurations. All previous lattice computations of the masses of charm baryons used gauge configurations produced with staggered sea quarks with a number of different actions for the valence quarks. In Refs. [40,41], a Clover charm valence quark was used on MILC $N_{f}=2+1$ gauge configurations at three values of the lattice spacing, $a=0.09$, $0.12,0.15$ fm. In Ref. [42], $N_{f}=2+1+1$ gauge configurations were produced using the highly improved staggered quark (HISQ) action. The valence light quark (up, down and strange) propagators are generated using the clover-impoved Wilson action. In order to reduce discretization artifacts, a relativistic heavy quark action was adopted for the charm quark. Finally, in Ref. [43], domain-wall fermions are used for the up, the down and the strange quarks on $N_{f}=2+1$ improved KogutSusskind sea quarks at one value of the lattice spacing, $a=0.12 \mathrm{fm}$. The relativistic Fermilab action was employed for the charm quark. We show the comparison of our results to those obtained in the aforementioned references in Fig. 18. Our results for $\Lambda_{c}, \Sigma_{c}, \Xi_{c c}, \Sigma_{c}^{*}$, $\Xi_{c c}^{*}$, and $\Omega_{c c c}$ baryons are extrapolated to the physical 

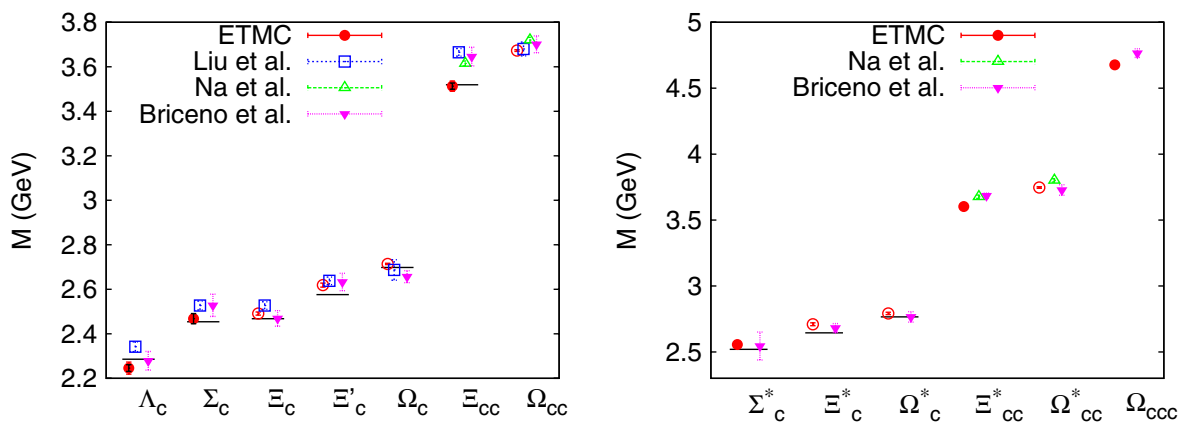

FIG. 18 (color online). Masses for charm baryons with spin-1/2 (left) and spin-3/2 (right) computed within lattice QCD, with the experimental values shown by the horizontal lines. Our results for $\Lambda_{c}, \Sigma_{c}, \Xi_{c c}, \Sigma_{c}^{*}, \Xi_{c c}^{*}$ and $\Omega_{c c c}$ baryons are extrapolated to the physical pion mass (shown with filled red circles), whereas for the rest we give the results obtained at $m_{\pi}=260 \mathrm{MeV}$ and $\beta=4.2$ (shown with open red circles). We include results obtained using a number of hybrid actions with staggered sea quarks from Refs. [43] (open blue squares), [40,41] (open green triangles) and [42] (filled magenta triangles).

pion mass using a polynomial fit with up $m_{\pi}^{3}$ terms. For the spin-1/2 $\Xi_{c}, \Xi_{c}^{\prime}, \Omega_{c}$, and $\Omega_{c c}$ baryons, we show the results obtained at $m_{\pi}=260 \mathrm{MeV}$, the smallest value of the pion mass considered in this work on the lattice with the smallest lattice spacing at $\beta=4.2$, for which cutoff effects are smallest. As can be seen, our results are in agreement with the results of the other studies except for the $\Xi_{c c}$ baryon, and with the experimental values. Although for the $\Xi_{c c}$ baryon we find a value consistent with the result of the SELEX experiment, one has to study the pion mass dependence in order to reach a final conclusion. In Fig. 18, we compare results for the spin-3/2 charm baryons. Our results for $\Xi_{c}^{*}, \Omega_{c}^{*}$, and $\Omega_{c c}^{*}$ baryons are obtained at $m_{\pi}=260 \mathrm{MeV}$ at $\beta=4.2$. There is good agreement among lattice results and with the known experimental values for $\Sigma_{c}^{*}, \Xi_{c}^{*}$, and $\Omega_{c}^{*}$ baryon masses. Thus, the lattice results can be taken as a prediction for the masses of the other charm spin-3/2 baryons shown in the figure.

\section{SUMMARY AND CONCLUSIONS}

In this work we have computed the strange and charm baryon masses using $N_{f}=2$ twisted mass fermions. For the strange and charm sector, we use Osterwalder-Seiler valence quarks. The bare strange and charm valence quark mass is tuned by requiring that the physical values of the mass of the kaon and $D$ meson be reproduced after the lattice results are extrapolated at the physical value of the pion mass.

We analyze gauge configurations for three values of the lattice spacings at the largest and smallest pion mass used in this study. We find that cutoff effects are small even in the case of the charm baryons. This is a somewhat surprising result, given that the Compton wavelength of the $D$-meson mass is of the same order as the lattice spacing.

Using simulations on two different volumes, we have obtained results that are consistent, showing that any volume effects are smaller than our statistical accuracy.
Another artifact of our lattice formulation is isospin breaking at finite lattice spacing. We have found that isospin breaking decreases with the lattice spacing, and it is consistent with zero for $a=0.056 \mathrm{fm}$, confirming the expected restoration of isospin symmetry.

Our results on the strange quark sector are consistent with recent results using Clover-improved fermions and domain-wall fermions on a staggered sea. There is an overall agreement also in thee case of the charm sector, where we compare our results to other studies that used staggered sea quarks. The overall consistence among lattice results, despite the different discretizations used, provides a strong validation of lattice QCD computations. Our results on the charm baryons reproduce the experimentally known values, and thus provide an estimate for the masses of the $\Omega_{c c}, \Xi_{c c}^{*}, \Omega_{c c}^{*}$ and $\Omega_{c c c}$ baryons.

\section{ACKNOWLEDGMENTS}

We would like to thank all members of ETMC for a very constructive and enjoyable collaboration. Numerical calculations have been performed at GENCI/IDRIS (Project i2011052271) and CC-IN2P3 computer centers. Computer time for this project was also made available to us by the John von Neumann Institute for Computing on the Jugene $\mathrm{BG} / \mathrm{P}$ system at the research center in Jülich and by the Computation-based Science and Technology Research Center of the Cyprus Institute through the infrastructure project Cy-Tera, cofunded by the European Regional Development Fund and the Republic of Cyprus through the Research Promotion Foundation (Project Cy-Tera NEA YПО $\Delta$ OMH/ $/ 2$ TPATH/0308/31). This work was partly supported by funding received from the Cyprus Research Promotion Foundation under Contracts No. PENEK/0609/17 and No. KY-ГА/0310/02. М. P. acknowledges financial support by the Marie Curie European Reintegration Grant of the 7th European Community Framework Programme under Contract No. PERG05-GA-2009-249309. M. G. was supported by the Marie Curie European training network ITN STRONGnet under Grant No. PITN-GA-2009-238353. 


\section{APPENDIX}

In Tables X, XI, and XII we give the strange octet baryon masses for $\beta=3.9, \beta=4.05$, and $\beta=4.2$, respectively and in Tables XIII, XIV, and XV the strange decuplet baryon masses. In Tables XVI, XVII, and XVIII we collect the charm octet baryon masses for the three beta and in Tables XIX, XX, and XXI the charm decuplet baryon masses. In Table XXII we give the masses for the spin-1/2 and spin-3/2 charm baryons with strangeness at $\beta=4.2$ at the lightest pion mass.

TABLE X. Strange octet baryon masses at $\beta=3.9$.

\begin{tabular}{|c|c|c|c|c|c|c|c|c|}
\hline$L / a$ & $a \mu_{h}$ & $a \mu_{l}$ & $a m_{\Sigma^{0}}$ & $a m_{\Sigma^{-}}$ & $a m_{\Sigma^{+}}$ & $a m_{\Xi^{0}}$ & $a m_{\Xi^{-}}$ & $a m_{\Lambda}$ \\
\hline 32 & 0.0217 & 0.0030 & $0.597(3)$ & $0.577(4)$ & $0.607(7)$ & $0.657(4)$ & $0.628(3)$ & $0.561(3)$ \\
\hline 24 & 0.0217 & 0.0040 & $0.610(5)$ & $0.600(3)$ & $0.622(4)$ & $0.671(3)$ & $0.638(2)$ & $0.577(4)$ \\
\hline 32 & 0.0217 & 0.0040 & $0.616(4)$ & $0.596(5)$ & $0.628(7)$ & $0.675(4)$ & $0.635(3)$ & $0.574(4)$ \\
\hline 24 & 0.0217 & 0.0064 & $0.628(5)$ & $0.610(6)$ & $0.640(9)$ & $0.687(5)$ & $0.650(4)$ & $0.602(4)$ \\
\hline 24 & 0.0217 & 0.0085 & $0.649(3)$ & $0.631(2)$ & $0.668(4)$ & $0.697(3)$ & $0.659(2)$ & $0.619(3)$ \\
\hline 24 & 0.0217 & 0.0100 & $0.654(4)$ & $0.643(5)$ & $0.666(6)$ & $0.696(5)$ & $0.668(3)$ & $0.633(4)$ \\
\hline 24 & 0.015 & 0.0064 & $0.596(9)$ & $0.585(6)$ & $0.633(9)$ & $0.658(11)$ & $0.597(12)$ & $0.588(4)$ \\
\hline 24 & 0.015 & 0.0085 & $0.641(5)$ & $0.598(11)$ & $0.663(6)$ & $0.674(5)$ & $0.621(6)$ & $0.594(9)$ \\
\hline 24 & 0.015 & 0.0100 & $0.635(7)$ & $0.627(8)$ & $0.645(15)$ & $0.659(14)$ & $0.622(7)$ & $0.614(6)$ \\
\hline 32 & 0.025 & 0.0040 & $0.623(5)$ & $0.606(5)$ & $0.633(9)$ & $0.687(6)$ & $0.651(4)$ & $0.587(8)$ \\
\hline 32 & 0.030 & 0.0040 & $0.645(4)$ & $0.630(5)$ & $0.664(6)$ & $0.729(6)$ & $0.688(4)$ & $0.606(5)$ \\
\hline 24 & 0.030 & 0.0064 & $0.654(5)$ & $0.640(6)$ & $0.674(7)$ & $0.732(5)$ & $0.682(7)$ & $0.624(4)$ \\
\hline 24 & 0.030 & 0.0085 & $0.688(5)$ & $0.651(9)$ & $0.703(6)$ & $0.739(6)$ & $0.706(4)$ & $0.626(9)$ \\
\hline
\end{tabular}

TABLE XI. Strange octet baryon masses at $\beta=4.05$.

\begin{tabular}{|c|c|c|c|c|c|c|c|c|}
\hline$L / a$ & $a \mu_{h}$ & $a \mu_{l}$ & $a m_{\Sigma^{0}}$ & $a m_{\Sigma^{-}}$ & $a m_{\Sigma^{+}}$ & $a m_{\Xi^{0}}$ & $a m_{\Xi^{-}}$ & $a m_{\Lambda}$ \\
\hline 32 & 0.0178 & 0.0030 & $0.477(5)$ & $0.470(8)$ & $0.488(9)$ & $0.519(6)$ & $0.504(4)$ & $0.455(5)$ \\
\hline 32 & 0.0178 & 0.0060 & $0.500(5)$ & $0.483(5)$ & $0.503(8)$ & $0.529(5)$ & $0.519(4)$ & $0.482(5)$ \\
\hline 32 & 0.0178 & 0.0080 & $0.512(4)$ & $0.506(5)$ & $0.522(4)$ & $0.541(4)$ & $0.527(3)$ & $0.496(5)$ \\
\hline 32 & 0.014 & 0.0030 & $0.463(5)$ & $0.456(9)$ & $0.474(9)$ & $0.496(6)$ & $0.482(4)$ & $0.445(6)$ \\
\hline 32 & 0.014 & 0.0060 & $0.495(4)$ & $0.473(6)$ & $0.497(8)$ & $0.513(6)$ & $0.500(4)$ & $0.479(4)$ \\
\hline 32 & 0.014 & 0.0080 & $0.494(8)$ & $0.508(9)$ & $0.508(5)$ & $0.512(7)$ & $0.507(3)$ & $0.485(6)$ \\
\hline 32 & 0.0166 & 0.0030 & $0.477(5)$ & $0.465(5)$ & $0.487(6)$ & $0.515(4)$ & $0.498(3)$ & $0.451(4)$ \\
\hline 32 & 0.0166 & 0.0060 & $0.496(5)$ & $0.483(5)$ & $0.502(5)$ & $0.526(4)$ & $0.511(4)$ & $0.480(5)$ \\
\hline 32 & 0.0166 & 0.0080 & $0.510(3)$ & $0.502(3)$ & $0.518(4)$ & $0.535(3)$ & $0.521(3)$ & $0.496(3)$ \\
\hline 32 & 0.019 & 0.0060 & $0.501(6)$ & $0.495(8)$ & $0.519(13)$ & $0.532(6)$ & $0.524(6)$ & $0.483(5)$ \\
\hline 32 & 0.020 & 0.0030 & $0.486(6)$ & $0.478(8)$ & $0.495(8)$ & $0.531(6)$ & $0.517(4)$ & $0.461(5)$ \\
\hline 32 & 0.020 & 0.0060 & $0.510(6)$ & $0.493(5)$ & $0.515(7)$ & $0.544(6)$ & $0.534(4)$ & $0.492(5)$ \\
\hline 32 & 0.020 & 0.0080 & $0.516(4)$ & $0.510(7)$ & $0.514(7)$ & $0.547(4)$ & $0.533(4)$ & $0.500(5)$ \\
\hline 32 & 0.025 & 0.0060 & $0.522(5)$ & $0.501(6)$ & $0.531(7)$ & $0.572(5)$ & $0.556(4)$ & $0.501(5)$ \\
\hline
\end{tabular}

TABLE XII. Strange octet baryon masses at $\beta=4.2$.

\begin{tabular}{|c|c|c|c|c|c|c|c|c|}
\hline$L / a$ & $a \mu_{h}$ & $a \mu_{l}$ & $a m_{\Sigma^{0}}$ & $a m_{\Sigma^{-}}$ & $a m_{\Sigma^{+}}$ & $a m_{\Xi^{0}}$ & $a m_{\Xi^{-}}$ & $a m_{\Lambda}$ \\
\hline 32 & 0.012 & 0.0065 & $0.402(4)$ & $0.396(4)$ & $0.405(5)$ & $0.418(4)$ & $0.406(4)$ & $0.392(4)$ \\
\hline 48 & 0.012 & 0.0020 & $0.362(3)$ & $0.360(3)$ & $0.368(4)$ & $0.390(2)$ & $0.381(2)$ & $0.344(3)$ \\
\hline 32 & 0.013 & 0.0065 & $0.406(7)$ & $0.405(7)$ & $0.417(7)$ & $0.430(6)$ & $0.416(6)$ & $0.395(7)$ \\
\hline 32 & 0.015 & 0.0065 & $0.411(4)$ & $0.409(4)$ & $0.413(4)$ & $0.431(4)$ & $0.421(4)$ & $0.397(4)$ \\
\hline 48 & 0.015 & 0.0020 & $0.374(2)$ & $0.371(3)$ & $0.380(3)$ & $0.404(2)$ & $0.397(2)$ & $0.352(2)$ \\
\hline 32 & 0.016 & 0.0065 & $0.417(7)$ & $0.409(4)$ & $0.413(4)$ & $0.442(5)$ & $0.421(4)$ & $0.397(4)$ \\
\hline
\end{tabular}


TABLE XIII. Strange decuplet baryon masses at $\beta=3.9$.

\begin{tabular}{|c|c|c|c|c|c|c|c|c|}
\hline$L / a$ & $a \mu_{h}$ & $a \mu_{l}$ & $a m_{\Sigma^{* 0}}$ & $a m_{\Sigma^{*-}}$ & $a m_{\Sigma^{*+}}$ & $a m_{\Xi^{* 0}}$ & $a m_{\Xi^{*-}}$ & $a m_{\Omega}$ \\
\hline 32 & 0.0217 & 0.0030 & $0.700(7)$ & $0.713(5)$ & $0.700(6)$ & $0.710(6)$ & $0.702(5)$ & $0.798(3)$ \\
\hline 32 & 0.0217 & 0.0040 & $0.726(4)$ & $0.718(6)$ & $0.715(6)$ & $0.739(4)$ & $0.721(4)$ & $0.809(3)$ \\
\hline 24 & 0.0217 & 0.0040 & $0.716(12)$ & $0.726(14)$ & $0.717(17)$ & $0.737(8)$ & $0.714(8)$ & $0.805(7)$ \\
\hline 24 & 0.0217 & 0.0064 & $0.749(7)$ & $0.749(7)$ & $0.740(11)$ & $0.754(8)$ & $0.734(8)$ & $0.815(6)$ \\
\hline 24 & 0.0217 & 0.0085 & $0.759(5)$ & $0.755(4)$ & $0.755(5)$ & $0.767(3)$ & $0.738(5)$ & $0.820(3)$ \\
\hline 24 & 0.0217 & 0.0100 & $0.773(8)$ & $0.772(8)$ & $0.773(9)$ & $0.784(5)$ & $0.749(7)$ & $0.834(4)$ \\
\hline 24 & 0.015 & 0.0064 & $0.737(24)$ & $0.736(7)$ & $0.746(7)$ & $0.728(15)$ & $0.708(10)$ & $0.772(16)$ \\
\hline 24 & 0.015 & 0.0085 & $0.751(10)$ & $0.736(20)$ & $0.755(7)$ & $0.723(11)$ & $0.659(18)$ & $0.771(9)$ \\
\hline 24 & 0.015 & 0.0100 & $0.730(18)$ & $0.757(19)$ & $0.746(16)$ & $0.720(15)$ & $0.667(10)$ & $0.771(11)$ \\
\hline 32 & 0.025 & 0.0040 & $0.730(9)$ & $0.723(12)$ & $0.710(14)$ & $0.747(12)$ & $0.738(8)$ & $0.827(7)$ \\
\hline 32 & 0.030 & 0.0040 & $0.724(11)$ & $0.743(10)$ & $0.739(9)$ & $0.782(7)$ & $0.765(8)$ & $0.870(6)$ \\
\hline 24 & 0.030 & 0.0064 & $0.772(6)$ & $0.769(6)$ & $0.760(11)$ & $0.797(7)$ & $0.781(6)$ & $0.874(6)$ \\
\hline 24 & 0.030 & 0.0085 & $0.782(11)$ & $0.736(7)$ & $0.746(7)$ & $0.812(7)$ & $0.751(11)$ & $0.771(9)$ \\
\hline
\end{tabular}

TABLE XIV. Strange decuplet baryon masses at $\beta=4.05$.

\begin{tabular}{|c|c|c|c|c|c|c|c|c|}
\hline$L / a$ & $a \mu_{h}$ & $a \mu_{l}$ & $a m_{\Sigma^{* 0}}$ & $a m_{\Sigma^{*-}}$ & $a m_{\Sigma^{*+}}$ & $a m_{\Xi^{* 0}}$ & $a m_{\Xi^{*-}}$ & $a m_{\Omega}$ \\
\hline 32 & 0.0178 & 0.0030 & $0.597(11)$ & $0.589(10)$ & $0.590(13)$ & $0.596(8)$ & $0.588(6)$ & $0.661(7)$ \\
\hline 32 & 0.0178 & 0.0060 & $0.593(8)$ & $0.586(8)$ & $0.582(8)$ & $0.582(7)$ & $0.583(7)$ & $0.638(6)$ \\
\hline 32 & 0.0178 & 0.0080 & $0.606(6)$ & $0.607(7)$ & $0.606(6)$ & $0.609(6)$ & $0.598(5)$ & $0.651(6)$ \\
\hline 32 & 0.014 & 0.0030 & $0.589(12)$ & $0.595(8)$ & $0.603(6)$ & $0.578(8)$ & $0.570(6)$ & $0.627(8)$ \\
\hline 32 & 0.014 & 0.0060 & $0.586(11)$ & $0.574(13)$ & $0.582(12)$ & $0.568(10)$ & $0.559(11)$ & $0.623(7)$ \\
\hline 32 & 0.014 & 0.0080 & $0.588(10)$ & $0.606(7)$ & $0.592(6)$ & $0.574(9)$ & $0.574(6)$ & $0.614(8)$ \\
\hline 32 & 0.0166 & 0.0030 & $0.570(12)$ & $0.578(7)$ & $0.561(8)$ & $0.573(6)$ & $0.567(6)$ & $0.630(5)$ \\
\hline 32 & 0.0166 & 0.0060 & $0.582(12)$ & $0.578(10)$ & $0.568(14)$ & $0.579(6)$ & $0.577(7)$ & $0.622(8)$ \\
\hline 32 & 0.0166 & 0.0080 & $0.615(4)$ & $0.609(6)$ & $0.604(7)$ & $0.601(5)$ & $0.586(5)$ & $0.648(4)$ \\
\hline 32 & 0.019 & 0.0060 & $0.592(16)$ & $0.576(13)$ & $0.551(19)$ & $0.579(8)$ & $0.583(9)$ & $0.648(5)$ \\
\hline 32 & 0.020 & 0.0030 & $0.611(7)$ & $0.605(8)$ & $0.610(7)$ & $0.606(7)$ & $0.596(8)$ & $0.671(5)$ \\
\hline 32 & 0.020 & 0.0060 & $0.599(10)$ & $0.591(9)$ & $0.595(11)$ & $0.594(11)$ & $0.591(9)$ & $0.657(6)$ \\
\hline 32 & 0.020 & 0.0080 & $0.610(6)$ & $0.619(6)$ & $0.605(6)$ & $0.613(8)$ & $0.597(6)$ & $0.660(5)$ \\
\hline 32 & 0.025 & 0.0060 & $0.598(11)$ & $0.589(12)$ & $0.577(15)$ & $0.613(7)$ & $0.613(8)$ & $0.674(8)$ \\
\hline
\end{tabular}

TABLE XV. Strange decuplet baryon masses at $\beta=4.2$.

\begin{tabular}{|c|c|c|c|c|c|c|c|c|}
\hline$L / a$ & $a \mu_{h}$ & $a \mu_{l}$ & $a m_{\Sigma^{* 0}}$ & $a m_{\Sigma^{*-}}$ & $a m_{\Sigma^{*+}}$ & $a m_{\Xi^{* 0}}$ & $a m_{\Xi^{*-}}$ & $a m_{\Omega}$ \\
\hline 32 & 0.012 & 0.0065 & $0.480(7)$ & $0.482(6)$ & $0.476(8)$ & $0.468(6)$ & $0.464(6)$ & $0.498(6)$ \\
\hline 48 & 0.012 & 0.0020 & $0.453(4)$ & $0.451(4)$ & $0.451(4)$ & $0.444(3)$ & $0.440(3)$ & $0.487(3)$ \\
\hline 32 & 0.013 & 0.0065 & $0.501(11)$ & $0.500(10)$ & $0.495(14)$ & $0.494(9)$ & $0.489(9)$ & $0.522(10)$ \\
\hline 32 & 0.015 & 0.0065 & $0.478(6)$ & $0.487(5)$ & $0.477(6)$ & $0.478(5)$ & $0.475(5)$ & $0.513(4)$ \\
\hline 48 & 0.015 & 0.0020 & $0.458(4)$ & $0.455(3)$ & $0.452(2)$ & $0.440(4)$ & $0.448(4)$ & $0.505(3)$ \\
\hline 32 & 0.016 & 0.0065 & $0.507(11)$ & $0.487(5)$ & $0.503(12)$ & $0.504(10)$ & $0.505(9)$ & $0.540(10)$ \\
\hline
\end{tabular}


TABLE XVI. Charm spin- $1 / 2$ baryon masses at $\beta=3.9$.

\begin{tabular}{|c|c|c|c|c|c|c|c|c|}
\hline$L / a$ & $a \mu_{h}$ & $a \mu_{l}$ & $a m_{\Sigma_{c}^{+}}$ & $a m_{\Sigma_{c}^{0}}$ & $a m_{\Sigma_{c}^{++}}$ & $a m_{\Xi_{c c}^{++}}$ & $a m_{\Xi_{c c}^{+}}$ & $a m_{\Lambda_{c}^{+}}$ \\
\hline 24 & 0.240 & 0.0040 & $1.100(6)$ & $1.105(13)$ & $1.102(14)$ & $1.532(5)$ & $1.528(4)$ & $1.015(8)$ \\
\hline 24 & 0.240 & 0.0064 & $1.117(6)$ & $1.059(20)$ & $1.122(6)$ & $1.552(3)$ & $1.533(4)$ & $1.045(5)$ \\
\hline 24 & 0.240 & 0.0085 & $1.131(6)$ & $1.125(5)$ & $1.135(5)$ & $1.555(4)$ & $1.541(5)$ & $1.063(3)$ \\
\hline 24 & 0.240 & 0.0100 & $1.139(4)$ & $1.131(5)$ & $1.138(5)$ & $1.559(3)$ & $1.551(2)$ & $1.070(3)$ \\
\hline 24 & 0.250 & 0.0040 & $1.128(7)$ & $1.120(9)$ & $1.099(14)$ & $1.591(5)$ & $1.575(5)$ & $1.055(6)$ \\
\hline 24 & 0.270 & 0.0040 & $1.154(5)$ & $1.157(5)$ & $1.161(6)$ & $1.640(4)$ & $1.628(3)$ & $1.069(4)$ \\
\hline 24 & 0.270 & 0.0064 & $1.172(7)$ & $1.164(5)$ & $1.176(7)$ & $1.648(5)$ & $1.634(4)$ & $1.096(5)$ \\
\hline 24 & 0.270 & 0.0085 & $1.192(4)$ & $1.181(4)$ & $1.188(5)$ & $1.658(3)$ & $1.651(3)$ & $1.115(3)$ \\
\hline 24 & 0.270 & 0.0100 & $1.196(4)$ & $1.189(4)$ & $1.197(4)$ & $1.660(3)$ & $1.649(25)$ & $1.122(3)$ \\
\hline 24 & 0.300 & 0.0040 & $1.203(9)$ & $1.214(5)$ & $1.221(5)$ & $1.736(6)$ & $1.731(4)$ & $1.135(4)$ \\
\hline 24 & 0.300 & 0.0064 & $1.227(4)$ & $1.162(22)$ & $1.236(5)$ & $1.747(4)$ & $1.722(6)$ & $1.148(5)$ \\
\hline 24 & 0.300 & 0.0085 & $1.244(4)$ & $1.236(4)$ & $1.244(4)$ & $1.759(3)$ & $1.736(6)$ & $1.169(3)$ \\
\hline 24 & 0.300 & 0.0100 & $1.251(3)$ & $1.237(5)$ & $1.249(4)$ & $1.762(2)$ & $1.751(3)$ & $1.172(3)$ \\
\hline
\end{tabular}

TABLE XVII. Charm spin-1/2 baryon masses at $\beta=4.05$.

\begin{tabular}{|c|c|c|c|c|c|c|c|c|}
\hline$L / a$ & $a \mu_{h}$ & $a \mu_{l}$ & $a m_{\Sigma_{c}^{+}}$ & $a m_{\Sigma_{c}^{0}}$ & $a m_{\Sigma_{c}^{++}}$ & $a m_{\Xi_{c c}^{++}}$ & $a m_{\Xi_{c c}^{+}}$ & $a m_{\Lambda_{c}^{+}}$ \\
\hline 32 & 0.170 & 0.0030 & $0.843(4)$ & $0.841(5)$ & $0.839(6)$ & $1.134(5)$ & $1.137(4)$ & $0.774(5)$ \\
\hline 32 & 0.170 & 0.0060 & $0.844(4)$ & $0.831(9)$ & $0.836(8)$ & $1.149(3)$ & $1.139(4)$ & $0.791(5)$ \\
\hline 32 & 0.170 & 0.0080 & $0.852(4)$ & $0.852(5)$ & $0.852(7)$ & $1.146(2)$ & $1.142(3)$ & $0.791(6)$ \\
\hline 32 & 0.200 & 0.0030 & $0.900(4)$ & $0.888(7)$ & $0.894(6)$ & $1.244(3)$ & $1.244(5)$ & $0.828(6)$ \\
\hline 32 & 0.200 & 0.0060 & $0.903(4)$ & $0.886(10)$ & $0.891(8)$ & $1.255(2)$ & $1.250(3)$ & $0.843(5)$ \\
\hline 32 & 0.200 & 0.0080 & $0.905(5)$ & $0.907(5)$ & $0.908(8)$ & $1.250(2)$ & $1.247(3)$ & $0.848(5)$ \\
\hline 32 & 0.210 & 0.0030 & $0.917(4)$ & $0.912(6)$ & $0.908(7)$ & $1.282(3)$ & $1.276(4)$ & $0.845(6)$ \\
\hline 32 & 0.210 & 0.0060 & $0.919(4)$ & $0.911(4)$ & $0.921(4)$ & $1.286(3)$ & $1.279(3)$ & $0.859(5)$ \\
\hline 32 & 0.210 & 0.0080 & $0.926(3)$ & $0.925(4)$ & $0.926(4)$ & $1.288(2)$ & $1.283(2)$ & $0.865(4)$ \\
\hline 32 & 0.230 & 0.0030 & $0.952(5)$ & $0.942(7)$ & $0.948(6)$ & $1.345(3)$ & $1.342(5)$ & $0.883(5)$ \\
\hline 32 & 0.230 & 0.0060 & $0.952(4)$ & $0.940(11)$ & $0.945(8)$ & $1.355(3)$ & $1.353(3)$ & $0.895(5)$ \\
\hline 32 & 0.230 & 0.0080 & $0.959(5)$ & $0.960(5)$ & $0.959(5)$ & $1.349(3)$ & $1.349(3)$ & $0.899(5)$ \\
\hline 32 & 0.260 & 0.0030 & $1.004(5)$ & $1.005(10)$ & $1.003(5)$ & $1.444(3)$ & $1.441(5)$ & $0.935(5)$ \\
\hline 32 & 0.260 & 0.0060 & $1.001(5)$ & $0.992(11)$ & $0.997(8)$ & $1.457(3)$ & $1.451(4)$ & $0.946(5)$ \\
\hline 32 & 0.260 & 0.0080 & $1.010(6)$ & $1.011(7)$ & $1.012(8)$ & $1.449(3)$ & $1.446(5)$ & $0.955(3)$ \\
\hline
\end{tabular}

TABLE XVIII. Charm spin- $1 / 2$ baryon masses at $\beta=4.2$.

\begin{tabular}{|c|c|c|c|c|c|c|c|c|}
\hline$L / a$ & $a \mu_{h}$ & $a \mu_{l}$ & $a m_{\Sigma_{c}^{+}}$ & $a m_{\Sigma_{c}^{0}}$ & $a m_{\Sigma_{c}^{++}}$ & $a m_{\Xi_{c c}^{++}}$ & $a m_{\Xi_{c c}^{+}}$ & $a m_{\Lambda_{c}^{+}}$ \\
\hline 32 & 0.130 & 0.0065 & $0.696(6)$ & $0.694(6)$ & $0.698(6)$ & $0.932(4)$ & $0.927(5)$ & $0.653(4)$ \\
\hline 32 & 0.160 & 0.0065 & $0.733(6)$ & $0.731(6)$ & $0.734(7)$ & $0.999(4)$ & $0.997(5)$ & $0.688(4)$ \\
\hline 32 & 0.185 & 0.0065 & $0.778(7)$ & $0.776(6)$ & $0.779(7)$ & $1.085(4)$ & $1.082(5)$ & $0.731(4)$ \\
\hline 32 & 0.210 & 0.0065 & $0.821(7)$ & $0.819(7)$ & $0.822(7)$ & $1.168(4)$ & $1.156(5)$ & $0.774(4)$ \\
\hline 48 & 0.136 & 0.0020 & $0.653(3)$ & $0.656(3)$ & $0.652(3)$ & $0.899(2)$ & $0.898(2)$ & $0.603(2)$ \\
\hline 48 & 0.170 & 0.0020 & $0.716(3)$ & $0.719(3)$ & $0.715(4)$ & $1.017(2)$ & $1.016(2)$ & $0.663(3)$ \\
\hline
\end{tabular}


TABLE XIX. Charm spin-3/2 baryon masses at $\beta=3.9$.

\begin{tabular}{|c|c|c|c|c|c|c|c|c|}
\hline$L / a$ & $a \mu_{h}$ & $a \mu_{l}$ & $a m_{\sum_{c}^{*+}}$ & $a m_{\sum_{c}^{* 0}}$ & $a m_{\sum_{c}^{*++}}$ & $a m_{\Xi_{c c}^{*++}}$ & $a m_{\Xi_{c c}^{*+}}$ & $a m_{\Omega_{c c c}}$ \\
\hline 24 & 0.240 & 0.0040 & $1.148(10)$ & $1.142(16)$ & $1.147(15)$ & $1.572(6)$ & $1.564(6)$ & $1.989(3)$ \\
\hline 24 & 0.240 & 0.0064 & $1.159(10)$ & $1.151(9)$ & $1.166(11)$ & $1.580(6)$ & $1.572(5)$ & $1.991(4)$ \\
\hline 24 & 0.240 & 0.0085 & $1.175(8)$ & $1.164(8)$ & $1.174(10)$ & $1.594(5)$ & $1.578(7)$ & $1.997(4)$ \\
\hline 24 & 0.240 & 0.0100 & $1.184(6)$ & $1.184(4)$ & $1.181(6)$ & $1.599(3)$ & $1.591(3)$ & $1.999(3)$ \\
\hline 24 & 0.250 & 0.0040 & $1.173(8)$ & $1.182(9)$ & $1.173(11)$ & $1.602(7)$ & $1.606(8)$ & $2.043(4)$ \\
\hline 24 & 0.270 & 0.0040 & $1.201(8)$ & $1.204(7)$ & $1.210(5)$ & $1.671(7)$ & $1.668(5)$ & $2.130(3)$ \\
\hline 24 & 0.270 & 0.0064 & $1.209(10)$ & $1.210(5)$ & $1.225(6)$ & $1.680(5)$ & $1.672(5)$ & $2.133(3)$ \\
\hline 24 & 0.270 & 0.0085 & $1.231(4)$ & $1.224(5)$ & $1.230(4)$ & $1.692(5)$ & $1.686(3)$ & $2.136(4)$ \\
\hline 24 & 0.270 & 0.0100 & $1.239(4)$ & $1.234(4)$ & $1.238(5)$ & $1.694(4)$ & $1.690(4)$ & $2.141(3)$ \\
\hline 24 & 0.300 & 0.0040 & $1.263(5)$ & $1.245(15)$ & $1.260(6)$ & $1.775(6)$ & $1.764(5)$ & $2.269(3)$ \\
\hline 24 & 0.300 & 0.0064 & $1.267(5)$ & $1.260(5)$ & $1.275(6)$ & $1.779(5)$ & $1.768(5)$ & $2.270(3)$ \\
\hline 24 & 0.300 & 0.0085 & $1.281(4)$ & $1.274(5)$ & $1.280(4)$ & $1.794(3)$ & $1.770(8)$ & $2.274(4)$ \\
\hline 24 & 0.300 & 0.0100 & $1.292(3)$ & $1.283(4)$ & $1.282(6)$ & $1.795(3)$ & $1.789(3)$ & $2.277(2)$ \\
\hline
\end{tabular}

TABLE XX. Charm spin-3/2 baryon masses at $\beta=4.05$.

\begin{tabular}{|c|c|c|c|c|c|c|c|c|}
\hline$L / a$ & $a \mu_{h}$ & $a \mu_{l}$ & $a m_{\sum_{c}^{*+}}$ & $a m_{\Sigma_{c}^{* 0}}$ & $a m_{\Sigma_{c}^{*++}}$ & $a m_{\Xi_{c c}^{*++}}^{*++}$ & $a m_{\Xi_{c c}^{*+}}$ & $a m_{\Omega_{c c c}}$ \\
\hline 32 & 0.170 & 0.0030 & $0.889(6)$ & $0.889(6)$ & $0.890(4)$ & $1.170(6)$ & $1.174(5)$ & $1.468(3)$ \\
\hline 32 & 0.170 & 0.0060 & $0.887(4)$ & $0.883(5)$ & $0.868(12)$ & $1.184(3)$ & $1.172(6)$ & $1.476(2)$ \\
\hline 32 & 0.170 & 0.0080 & $0.885(5)$ & $0.890(7)$ & $0.884(5)$ & $1.176(3)$ & $1.175(4)$ & $1.468(3)$ \\
\hline 32 & 0.200 & 0.0030 & $0.940(6)$ & $0.940(6)$ & $0.941(4)$ & $1.277(4)$ & $1.281(4)$ & $1.616(3)$ \\
\hline 32 & 0.200 & 0.0060 & $0.928(6)$ & $0.937(4)$ & $0.918(11)$ & $1.288(3)$ & $1.281(3)$ & $1.623(2)$ \\
\hline 32 & 0.200 & 0.0080 & $0.936(5)$ & $0.945(6)$ & $0.935(5)$ & $1.279(3)$ & $1.281(3)$ & $1.612(3)$ \\
\hline 32 & 0.210 & 0.0030 & $0.956(6)$ & $0.956(6)$ & $0.958(4)$ & $1.310(4)$ & $1.314(4)$ & $1.663(3)$ \\
\hline 32 & 0.210 & 0.0060 & $0.955(5)$ & $0.954(4)$ & $0.948(5)$ & $1.316(3)$ & $1.310(4)$ & $1.667(2)$ \\
\hline 32 & 0.210 & 0.0080 & $0.957(4)$ & $0.962(4)$ & $0.958(4)$ & $1.316(3)$ & $1.313(3)$ & $1.663(3)$ \\
\hline 32 & 0.230 & 0.0030 & $0.989(6)$ & $0.989(6)$ & $0.992(4)$ & $1.376(4)$ & $1.379(4)$ & $1.761(3)$ \\
\hline 32 & 0.230 & 0.0060 & $0.984(4)$ & $0.987(4)$ & $0.967(11)$ & $1.387(3)$ & $1.380(4)$ & $1.768(2)$ \\
\hline 32 & 0.230 & 0.0080 & $0.986(5)$ & $0.995(6)$ & $0.986(5)$ & $1.378(3)$ & $1.380(3)$ & $1.758(3)$ \\
\hline 32 & 0.260 & 0.0030 & $1.039(6)$ & $1.038(6)$ & $1.036(7)$ & $1.473(4)$ & $1.476(4)$ & $1.905(3)$ \\
\hline 32 & 0.260 & 0.0060 & $1.033(5)$ & $1.032(5)$ & $1.016(11)$ & $1.485(3)$ & $1.478(4)$ & $1.910(3)$ \\
\hline 32 & 0.260 & 0.0080 & $1.034(5)$ & $1.039(6)$ & $1.032(10)$ & $1.472(3)$ & $1.472(5)$ & $1.898(4)$ \\
\hline
\end{tabular}

TABLE XXI. Charm spin-3/2 baryon masses at $\beta=4.05$.

\begin{tabular}{|c|c|c|c|c|c|c|c|c|}
\hline$L / a$ & $a \mu_{h}$ & $a \mu_{l}$ & $a m_{\sum_{c}^{*+}}$ & $a m_{\sum_{c}^{* 0}}$ & $a m_{\Sigma_{c}^{*++}}$ & $a m_{\Xi_{c c}^{*++}}$ & $a m_{\Xi_{c c}^{*+}}$ & $a m_{\Omega_{c c c}}$ \\
\hline 32 & 0.130 & 0.0065 & $0.730(8)$ & $0.730(8)$ & $0.727(8)$ & $0.958(6)$ & $0.963(6)$ & $1.191(3)$ \\
\hline 32 & 0.160 & 0.0065 & $0.763(8)$ & $0.763(8)$ & $0.761(8)$ & $1.025(6)$ & $1.029(6)$ & $1.287(3)$ \\
\hline 32 & 0.185 & 0.0065 & $0.805(8)$ & $0.804(8)$ & $0.802(8)$ & $1.107(6)$ & $1.109(6)$ & $1.408(3)$ \\
\hline 32 & 0.210 & 0.0065 & $0.845(9)$ & $0.845(8)$ & $0.843(8)$ & $1.188(6)$ & $1.193(6)$ & $1.526(3)$ \\
\hline 48 & 0.136 & 0.0020 & $0.686(4)$ & $0.688(3)$ & $0.683(4)$ & $0.925(2)$ & $0.926(2)$ & $1.166(1)$ \\
\hline 48 & 0.170 & 0.0020 & $0.744(4)$ & $0.746(3)$ & $0.741(4)$ & $1.039(2)$ & $1.039(2)$ & $1.333(1)$ \\
\hline
\end{tabular}

TABLE XXII. Strange charm spin- $1 / 2$ and spin- $3 / 2$ baryon masses at $\beta=4.2$ at the tuned heavy quark masses.

\begin{tabular}{|c|c|c|c|c|c|c|c|c|c|c|}
\hline$L / a$ & $a \mu_{s}$ & $a \mu_{c}$ & $a \mu_{l}$ & $a m_{\Xi_{c}}$ & $a m_{\Xi_{c}^{\prime}}$ & $a m_{\Omega_{c}}$ & $a m_{\Omega_{c c}}$ & $a m_{\Xi_{c}^{*}}$ & $a m_{\Omega_{c}^{*}}$ & $a m_{\Omega_{c c}^{*}}$ \\
\hline 48 & 0.015 & 0.17 & 0.0020 & $0.708(2)$ & $0.745(3)$ & $0.771(2)$ & $1.044(1)$ & $0.770(3)$ & $0.794(2)$ & $1.065(2)$ \\
\hline
\end{tabular}


[1] C. Alexandrou et al. (ETM Collaboration), Phys. Rev. D 78, 014509 (2008).

[2] C. Alexandrou, R. Baron, J. Carbonell, V. Drach, P. Guichon, K. Jansen, T. Korzec, and O. Pène (ETM Collaboration), Phys. Rev. D 80, 114503 (2009).

[3] S. Aoki et al. (PACS-CS Collaboration), Phys. Rev. D 79, 034503 (2009).

[4] S. Durr et al., Science 322, 1224 (2008).

[5] A. Walker-Loud, H.-W. Lin, D. Richards, R. Edwards, M. Engelhardt et al., Phys. Rev. D 79, 054502 (2009).

[6] M. Mattson et al. (SELEX Collaboration), Phys. Rev. Lett. 89, 112001 (2002).

[7] J. Russ (SELEX Collaboration), arXiv:hep-ex/0209075.

[8] A. Ocherashvili et al. (SELEX Collaboration), Phys. Lett. B 628, 18 (2005).

[9] B. Aubert et al. (BABAR Collaboration), Phys. Rev. D 74, 011103 (2006).

[10] S. Ratti, Nucl. Phys. B, Proc. Suppl. 115, 33 (2003).

[11] R. Chistov et al. (Belle Collaboration), Phys. Rev. Lett. 97, 162001 (2006).

[12] C. W. Bernard, T. Burch, K. Orginos, D. Toussaint, T. DeGrand, C. DeTar, S. Datta, S. Gottlieb, U. Heller, and R. Sugar, Phys. Rev. D 64, 054506 (2001).

[13] C. Aubin, C. Bernard, C. DeTar, J. Osborn, S. Gottlieb, E. Gregory, D. Toussaint, U. Heller, J. Hetrick, and R. Sugar, Phys. Rev. D 70, 094505 (2004).

[14] A. Bazavov et al. (MILC collaboration), Phys. Rev. D 82, 074501 (2010).

[15] B. Blossier et al. (ETM Collaboration), J. High Energy Phys. 04 (2008) 020.

[16] B. Blossier et al. (ETM Collaboration), J. High Energy Phys. 07 (2009) 043.

[17] P. Boucaud et al. (ETM Collaboration), Phys. Lett. B 650, 304 (2007).

[18] P. Boucaud et al. (ETM Collaboration), Comput. Phys. Commun. 179, 695 (2008).

[19] R. Frezzotti and G. Rossi, Proc. Sci. LATTICE (2007) 277.

[20] P. Dimopoulos et al. (ETM Collaboration), Proc. Sci. LATTICE (2008) 103.

[21] K. Jansen, Proc. Sci. LATTICE (2008) 010.

[22] P. Weisz, Nucl. Phys. B212, 1 (1983).
[23] P. Boucaud et al., Comput. Phys. Commun. 179, 695 (2008).

[24] R. Frezzotti, G. Martinelli, M. Papinutto, and G. Rossi, J. High Energy Phys. 04 (2006) 038.

[25] R. Frezzotti and G. Rossi, J. High Energy Phys. 08 (2004) 007.

[26] R. Frezzotti and G. Rossi, J. High Energy Phys. 10 (2004) 070.

[27] A. M. Abdel-Rehim, R. Lewis, R. M. Woloshyn, and J. M. S. Wu, Eur. Phys. J. A 31, 773 (2007).

[28] A. M. Abdel-Rehim, R. Lewis, R. M. Woloshyn, and J. M. S. Wu, Phys. Rev. D 74, 014507 (2006).

[29] C. Alexandrou, M. Brinet, J. Carbonell, M. Constantinou, P. Harraud, P. Guichon, K. Jansen, T. Korzec, and M. Papinutto (ETM Collaboration), Phys. Rev. D 83, 045010 (2011).

[30] R. Baron et al. (ETM Collaboration), J. High Energy Phys. 08 (2010) 097.

[31] B. L. Ioffe, Nucl. Phys. B188, 317 (1981).

[32] D. B. Leinweber, R. M. Woloshyn, and T. Draper, Phys. Rev. D 43, 1659 (1991).

[33] D. B. Leinweber, T. Draper, and R. M. Woloshyn, Phys. Rev. D 46, 3067 (1992).

[34] U. Wolff (ALPHA Collaboration), Comput. Phys. Commun. 156, 143 (2004).

[35] M. M. Nagels, Th. A. Rijken, J. J. De Swart, G. C. Oades, J. L. Petersen, A.C. Irving, C. Jarlskog, W. Pfeil, H. Pilkuhn, and H. P. Jakob, Nucl. Phys. B147, 189 (1979).

[36] M. M. Nagels, T. A. Rijken, and J. J. de Swart, Phys. Rev. D 20, 1633 (1979).

[37] B. C. Tiburzi and A. Walker-Loud, Phys. Lett. B 669, 246 (2008).

[38] B. C. Tiburzi and A. Walker-Loud, Nucl. Phys. A764, 274 (2006).

[39] W. Bietenholz, V. Bornyakov, M. Gockeler, R. Horsley, W. Lockhart et al., Phys. Rev. D 84, 054509 (2011).

[40] H. Na and S. Gottlieb, Proc. Sci. LATTICE (2007) 124.

[41] H. Na and S. Gottlieb, Proc. Sci. LATTICE (2008) 119.

[42] R. A. Briceno, D. Bolton, and H.-W. Lin, Proc. Sci. LATTICE (2011) 116.

[43] L. Liu, H.-W. Lin, K. Orginos, and A. Walker-Loud, Phys. Rev. D 81, 094505 (2010). 